\title{
Affine schemes and topological closures in the Zariski-Riemann space of valuation rings
}

\author{
Bruce Olberding \\ Department of Mathematical Sciences, New Mexico State University, Las Cruces, NM 88003-8001
}

\begin{abstract}
Let $F$ be a field, let $D$ be a subring of $F$, and let $\mathfrak{X}$ be the Zariski-Riemann space of valuation rings containing $D$ and having quotient field $F$. We consider the Zariski, inverse and patch topologies on $\mathfrak{X}$ when viewed as a projective limit of projective integral schemes having function field contained in $F$, and we characterize the locally ringed subspaces of $\mathfrak{X}$ that are affine schemes.
\end{abstract}

Keywords: Zariski-Riemann space, valuation ring, projective model, spectral space, Prüfer domain

2010 MSC: 13A18, 13B22, 13F05, 14A15

\section{Introduction}

Throughout this article, $F$ is a field, $D$ is a subring of $F$ and $\mathfrak{X}$ denotes the collection of all valuation rings between $D$ and $F$ having quotient field $F$. For each subset $S$ of $F$, denote by $\mathfrak{X}_{S}$ the set of all valuation rings in $\mathfrak{X}$ containing $S$. The set $\mathfrak{X}$ is endowed with the topology whose basic open sets are of the form $\mathfrak{X}_{S}$, where $S$ is a finite subset of $F$. The topological space $\mathfrak{X}$ is the Zariski-Riemann space of $F / D$ (sometimes called the generalized Riemann manifold, Riemann-Zariski space, Zariski-Riemann manifold, abstract Riemann surface, or Riemann variety), and the topology thus defined is the Zariski topology on $\mathfrak{X}$. Zariski showed that $\mathfrak{X}$ is quasicompact and used this as a step in the proof of resolution of singularities of algebraic surfaces in characteristic 0 by reducing an infinite resolving system to a finite one [44]. But $\mathfrak{X}$ itself can be viewed as a geometric object. It is a locally ringed space with structure sheaf $\mathcal{O}_{\mathfrak{X}}$ defined by $\mathcal{O}_{\mathfrak{X}}(U)=\bigcap_{V \in U} V$ for each nonempty open subset $U$ of $\mathfrak{X}$. Moreover, as a locally ringed space, $\mathfrak{X}$ is the projective limit of the projective models of $F / D$, those projective integral schemes over $\operatorname{Spec}(D)$ whose function field is a subfield of $F$. Thus, while generally not a scheme, $\mathfrak{X}$ is a projective limit of projective schemes, and the valuation rings in $\mathfrak{X}$ can be used to track generic points of closed subschemes in blowups of projective models of $F / D$; this is the point of view taken in Zariski's theory of birational correspondence 43.

The aim of this article is to develop from a basic point of view some of the topological features of the Zariski-Riemann space as a projective limit of projective models. We often

Email address: olberdin@nmsu.edu (Bruce Olberding) 
do not assume anything more about $D$ other than that it is a subring of $F$, and sometimes that $\operatorname{Spec}(D)$ is a Noetherian space, so as to assure that projective models of $F / D$ are Noetherian spectral spaces. The focus is the interplay between the topology of $\mathfrak{X}$ and that of the projective models of $F / D$. By the "topology" of a subspace $Z$ of $\mathfrak{X}$ we mean not only the topology on $Z$ induced by the Zariski topology, but the patch and inverse topologies also. In this way, our approach to the topological nature of $\mathfrak{X}$ is influenced by the article [16], where the authors consider these three topologies also. Whereas in [16] the authors use the patch topology (and a nice interpretation of the patch topology in terms of ultrafilter limits of valuation rings; see Remark 3.9 (1) below) as a unifying theme, our preference is for the inverse topology because of its application to classifying affine schemes in $\mathfrak{X}$ (see Section 6), as well as for describing irredundant representations, as is done in 34]. However, as noted in Proposition 2.1 and in [16, Remark 2.2], inverse closure is simply the composition of patch closure with closure under generalizations. As in [16], we also emphasize the Kronecker function ring construction from multiplicative ideal theory for representing $\mathfrak{X}$ as the prime spectrum of a ring. In particular, in Section 4 we use this construction to exhibit an affine scheme that maps onto $\mathfrak{X}$ via a morphism of locally ringed spaces that is, by a theorem of Dobbs and Fontana, a homeomorphism on the underlying spaces. The ring of global sections of this scheme is a Prüfer domain that encodes the valuation theory of $\mathfrak{X}$ into the prime spectrum of a ring. In particular, as proved in [9] and [10], $\mathfrak{X}$ is a spectral space.

In Section 2 we develop some basic properties regarding patch closure and inverse closure in a spectral space. In Section 3, we discuss Zariski's representation of $\mathfrak{X}$ as a projective limit of projective models. One of the main consequences that we draw from this is that when the base ring $D$ is Noetherian, then $\mathfrak{X}$ is a projective limit of Noetherian spectral spaces. As discussed in Section 2, the patch and inverse closures are more transparent on Noetherian spectral spaces, so this representation of $\mathfrak{X}$ as a projective limit is helpful for clarifying the patch and inverse closures; in particular, in Corollary 3.4. we show how patch and inverse closures are determined by the images of the domination maps from $\mathfrak{X}$ to the approximating projective models. As an application, we describe some patch dense subsets of $\mathfrak{X}$. In Section 5, we take a different point of view and describe the inverse closure of a subset of $\mathfrak{X}$ in terms of its image in the affine scheme represented by the prime spectrum of the Kronecker function ring of $F / D$. This allows us to characterize in Theorem 6.1 the subspaces of $\mathfrak{X}$ that are affine schemes when viewed with the locally ringed space structure inherited from $\mathfrak{X}$.

While this article is focused on the topology of the Zariski-Riemann space, in future articles we apply these ideas to describing properties of irredundant intersections of valuation rings and the geometry of integrally closed rings.

Conventions. All rings are commutative and contain an identity. An overring of a domain $R$ is a ring between $R$ and its quotient field. The set of prime ideals of the ring $R$ is denoted $\operatorname{Spec}(R)$; the set of maximal ideals by $\operatorname{Max}(R)$. When relevant, $\operatorname{Spec}(R)$ denotes not only a set but an affine scheme, and sometimes a submodel of a projective model, but these different uses should always be clear from context. As noted above, we write $\mathfrak{X}_{R}$ for $\{V \in \mathfrak{X}: R \subseteq V\}$, so that $\mathfrak{X}_{R}$ is the subspace of $\mathfrak{X}$ consisting of the valuation rings in $\mathfrak{X}$ between $R$ and $F$. As discussed in Section 3, we do not assume that a projective model of $F / D$ has function field $F$. Explanations of other variations on traditional terminology can be found in the following places: generic point of a not-necessarily-closed subset of a 
spectral space (after Corollary 2.3); affine subset of $\mathfrak{X}$ (beginning of $\S 4$ ); dominant system of projective models (end of $\S 3$ ); $X(Z)=$ the image of a subset $Z$ of $\mathfrak{X}$ in the projective model $X(\S 3)$.

\section{Spectral spaces}

A topological space $X$ is a spectral space if $X$ is quasicompact and $T_{0}$; the quasicompact open subsets of $X$ are closed under finite intersection and form an open basis; and every nonempty irreducible closed subset of $X$ has a generic point. By a theorem of Hochster, these are precisely the topological spaces which arise as the prime spectrum of a commutative ring [24]. As we recall in the next section, $\mathfrak{X}$ and the projective models of $F / D$ are spectral spaces. Thus the topological notions developed in this section for spectral spaces will apply to these two cases. Although we consider several topologies on a spectral space, to minimize confusion we introduce notation for operators which we use to distinguish certain subsets of $X$. Where possible we use these operators rather than shift between topologies. These operators are defined in terms of standard topological notions on $X$.

$$
\begin{aligned}
\operatorname{cl}(Y)= & \text { intersection of all closed sets containing } Y . \\
\operatorname{inv}(Y)= & \text { intersection of all quasicompact open sets containing } Y . \\
\operatorname{gen}(Y)= & \text { intersection of all open sets containing } Y . \\
\operatorname{patch}(Y)= & \text { intersection of all subsets between } Y \text { and } X \text { of the form } U_{1} \cup\left(X \backslash U_{2}\right), \\
& \text { where } U_{1}, U_{2} \text { are quasicompact open sets of } X . \\
\operatorname{pt}(Y)= & \text { the points of } \operatorname{inv}(Y) \text { closed in the subspace topology. }
\end{aligned}
$$

Thus $\operatorname{cl}(Y)$ is the closure of $Y$ in $X$. Both $\operatorname{inv}(Y)$ and $\operatorname{patch}(Y)$ can also be interpreted as closures in appropriate topologies; namely, the subset $Y$ of $X$ is patch closed if $Y=\operatorname{patch}(Y)$; $Y$ is inverse closed if $Y=\operatorname{inv}(Y)$. The inverse topology on the spectral space $X$ is the topology whose closed sets are the inverse closed subsets of $X$, while the patch topology on $X$ has for closed sets the patch closed subsets of $X$. The patch topology gives $X$ the structure of a zero-dimensional compact Hausdorff space [26, p. 72]. The set gen $(Y)$ is the closure of $Y$ under generalizations, and while it too defines a topology, we will not have occasion to use this topology. (Recall that if $x, y \in X$ and $x \in \operatorname{cl}(\{y\})$, then $y$ is a generalization of $x$ and $x$ is a specialization of $y$.)

A subset $Y$ of $X$ is inverse open if its complement in $X$ is inverse closed; $Y$ is patch open if its complement in $X$ is patch closed. It is clear that closed subsets of $X$ and inverse closed subsets of $X$ are patch closed, and hence the patch topology refines the spectral topology on $X$ and its inverse topology. Both of these topologies were used by Hochster in 24], who proved that the inverse and patch topologies are again spectral. (Hochster did not give a name to the inverse topology; this terminology was evidently introduced by Schwartz in 37].) More precisely, let $Y$ and $X$ be spectral spaces. Then a map $f: Y \rightarrow X$ is spectral if it is continuous and the preimages of quasicompact open subsets are quasicompact. The patch closed subsets of $X$ are precisely those subsets $Y$ of $X$ that are spectral in the subspace topology and for which $Y \cap U$ is quasicompact in $Y$ for all quasicompact open subsets $U$ of $X$ (i.e., the inclusion mapping $Y \rightarrow X$ is a spectral map) [24, p. 45]. 
Proposition 2.1. The following statements hold for a subset $Y$ of the spectral space $X$.

(1) $\operatorname{inv}(Y)$ and $\operatorname{patch}(Y)$ are spectral spaces in the subspace topology of $X$.

(2) $\operatorname{gen}(Y) \subseteq \operatorname{inv}(Y)$ and $\operatorname{pt}(Y) \subseteq \operatorname{patch}(Y) \subseteq \operatorname{inv}(Y) \cap \operatorname{cl}(Y)$.

(3) $\operatorname{inv}(Y)=\operatorname{gen}(\operatorname{pt}(Y))=\operatorname{gen}(\operatorname{patch}(Y))$.

Proof. That patch $(Y)$ is a spectral space in the subspace topology is discussed above. Since an inverse closed subset is patch closed, it follows that $\operatorname{inv}(Y)$ is also a spectral space in the subspace topology. To prove (2), first observe that it is clear from the relevant definitions that $\operatorname{gen}(Y) \subseteq \operatorname{inv}(Y)$ and $\operatorname{patch}(Y) \subseteq \operatorname{inv}(Y) \cap \operatorname{cl}(Y)$. We claim that $\operatorname{pt}(Y) \subseteq \operatorname{patch}(Y)$. Since $\operatorname{patch}(Y) \subseteq \operatorname{inv}(Y)$ and $\operatorname{inv}(Y)$ is patch closed, it suffices to show that $\operatorname{pt}(Y)$ is a subset of the patch closure of $Y \operatorname{in} \operatorname{inv}(Y)$. Let $U$ be a quasicompact open subset of $\operatorname{inv}(Y)$, and let $V$ be the complemement of a quasicompact open subset of $\operatorname{inv}(Y)$. Since $\operatorname{inv}(Y)$ is quasicompact (this follows from (1)), so is the closed subset $V$, and hence $U \cup V$, as a union of two quasicompact subsets, is quasicompact. Since $Y \subseteq U \cup V \subseteq \operatorname{inv}(Y)$, it follows that $U \cup V$ meets every inverse open subset of $\operatorname{inv}(Y)$. Each $x \in \operatorname{pt}(Y)$ is a closed point in $\operatorname{inv}(Y)$, so $\{x\}$ is an intersection of complements of quasicompact open subsets of $\operatorname{inv}(Y)$. However, the intersection of finitely many of any of these inverse open (and spectral closed) subsets meets $U \cup V$, so since $U \cup V$ is quasicompact, this forces $x \in U \cup V$, proving that $\operatorname{pt}(Y) \subseteq \operatorname{patch}(Y)$. This proves $(2)$.

To prove (3), note that $\operatorname{since} \operatorname{inv}(Y)$ is a spectral space, then for each $y \in \operatorname{inv}(Y)$, there exists a closed point $x \in \operatorname{inv}(Y)$ such that $x \in \operatorname{cl}(\{y\})$. Hence $\operatorname{inv}(Y) \subseteq \operatorname{gen}(\operatorname{pt}(Y))$, and since the reverse inclusion is clear, $\operatorname{inv}(Y)=\operatorname{gen}(\operatorname{pt}(Y))$. Moreover, since by $(2)$, $\operatorname{pt}(Y) \subseteq \operatorname{patch}(Y) \subseteq \operatorname{inv}(Y)$, it follows then that $\operatorname{inv}(Y)=\operatorname{gen}(\operatorname{patch}(Y))$.

That $\operatorname{inv}(Y)=\operatorname{gen}(\operatorname{patch}(Y))$ is also noted in [16, Remark 2.2].

If $Y$ is a subspace of the spectral space $X$ and not every point in $Y$ is closed, then $Y \nsubseteq \operatorname{pt}(Y)$. However, when $Y$ is quasicompact, then $\operatorname{pt}(Y) \subseteq Y$, and also in this case, $\operatorname{inv}(Y)$ collapses to gen $(Y)$ :

Proposition 2.2. The following are equivalent for a subspace $Y$ of the spectral space $X$.

(1) $Y$ is quasicompact.

(2) $\operatorname{inv}(Y)=\operatorname{gen}(Y)$.

(3) $\operatorname{pt}(Y) \subseteq Y$.

Proof. (1) $\Rightarrow$ (2) By Proposition 2.1 (2), gen $(Y) \subseteq \operatorname{inv}(Y)$. Suppose $x \in \operatorname{inv}(Y) \backslash \operatorname{gen}(Y)$. Then $\operatorname{cl}(\{x\})$ does not meet $Y$, so that $Y$ is an open subset of the quasicompact space $Y \cup\{x\}$. As such, $Y=\bigcup_{\alpha}\left((Y \cup\{x\}) \cap U_{\alpha}\right)$, where $\left\{U_{\alpha}\right\}$ is a collection of quasicompact open subsets of $X$. Since $Y$ is quasicompact, it follows that $Y=(Y \cup\{x\}) \cap U$, where $U$ is a quasicompact open subset of $X$. But then $Y \subseteq U$ and $x \notin U$, in contradiction to the assumption that $x \in \operatorname{inv}(Y)$. Thus $\operatorname{inv}(Y)=\operatorname{gen}(Y)$.

$(2) \Rightarrow(3)$ Since by $(2), \operatorname{inv}(Y) \subseteq \operatorname{gen}(Y)$, it follows that if $x \in \operatorname{pt}(Y)$, then there exists $y \in Y$ such that $y \in \operatorname{cl}(\{x\})$. But since $x$ is a closed point in $\operatorname{inv}(Y)$, this forces $x=y \in Y$.

$(3) \Rightarrow(1)$ Assuming (3), Proposition 2.1(2) implies $\operatorname{inv}(Y)=\operatorname{gen}(\operatorname{pt}(Y)) \subseteq \operatorname{gen}(Y) \subseteq$ $\operatorname{inv}(Y)$, and hence $\operatorname{inv}(Y)=\operatorname{gen}(Y)$. Schwartz and Tressl show in [38, Proposition 2.3] that 
$Y$ is quasicompact if and only if $\operatorname{gen}(Y)$ is patch closed. Thus since $\operatorname{inv}(Y)$ is patch closed, we conclude that $\operatorname{gen}(Y)$ is patch closed, and hence $Y$ is quasicompact.

Corollary 2.3. A spectral space $X$ is Noetherian if and only if $\operatorname{inv}(Y)=\operatorname{gen}(Y)$ for all (open) subsets $Y$ of $X$.

Proof. If $X$ is Noetherian, then so is any subspace $Y$ of $X$, and hence $Y$ is quasicompact and by the proposition satisfies $\operatorname{inv}(Y)=\operatorname{gen}(Y)$. Conversely, if $\operatorname{inv}(Y)=\operatorname{gen}(Y)$ for all open subsets $Y$ of $X$, then by the proposition, each open subset is quasicompact. This then implies that $X$ is a Noetherian space.

Remark 2.4. Let $Y$ be a subspace of the spectral space $X$. The fact that $\operatorname{inv}(Y)=$ gen $(Y)$ when $Y$ is quasicompact is a slightly stronger version of [16, Proposition 2.6] and 38, Proposition 2.3], where it is shown that the generic closure of a quasicompact set is patch closed. A subspace $Y$ of $X$ is quasicompact in the inverse topology if and only if $\operatorname{cl}(Y)=\bigcup_{y \in Y} \operatorname{cl}(\{y\})$ [38, Corollary 2.4].

In Section 5 we show that an inverse closed subspace of $\mathfrak{X}$ admits the structure of a locally ringed space, a fact which ultimately depends on the following proposition.

Proposition 2.5. An inverse closed subspace of an irreducible spectral space is irreducible.

Proof. Let $Y$ be an inverse closed subspace of the irreducible spectral space $X$. Let $U$ and $V$ be open sets of $X$ such that $U \cap Y$ and $V \cap Y$ are nonempty. Since $X$ is a spectral space, $X$ has a basis of quasicompact open subsets, so we may assume that $U$ and $V$ are quasicompact open subsets of $X$. Since $Y$ is inverse closed in $X$, there is a collection $\left\{U_{\alpha}\right\}$ of quasicompact open subsets of $X$ such that $Y=\bigcap_{\alpha} U_{\alpha}$. Since $X$ is irreducible, the intersection of any two nonempty open subsets in $X$ is nonempty, and hence the collection $\{U, V\} \cup\left\{U_{\alpha}\right\}$ has the finite intersection property. Since a spectral space with the inverse topology is again a spectral space, then $X$ is quasicompact in the inverse topology. Therefore, the intersection of inverse closed sets $Y=U \cap V \cap\left(\bigcap_{\alpha} U_{\alpha}\right)$ is nonempty, which proves that $Y$ is irreducible.

When $Y$ is a Noetherian subspace of $X$, then the patch closure can be interpreted in terms of generic points of subsets of $Y$. We say that $x$ is a generic point for a subset $Y$ of $X$ if $\operatorname{cl}(\{x\})=\operatorname{cl}(Y)$; i.e., $x$ is the generic point of the closed set $\operatorname{cl}(Y)$. Recall that a subset $Y$ of a topological space $X$ is retrocompact if $Y \cap U$ is quasicompact for every quasicompact open subset $U$ of $X$ (i.e., the inclusion map $Y \rightarrow X$ is a spectral map).

Proposition 2.6. Let $Y$ be a subset of the spectral space $X$.

(1) If $x \in X$ and $x$ is a generic point for some subset of $Y$, then $x \in \operatorname{patch}(Y)$.

(2) If $Y$ is retrocompact (which is the case if $Y$ is a Noetherian subspace of $X$ ), then

$$
\operatorname{patch}(Y)=\{x \in X: x \text { is a generic point for some subset of } Y\} \text {. }
$$

Proof. (1) Suppose that $x \in X$ and $\operatorname{cl}(\{x\})=\operatorname{cl}(Z)$ for some subset $Z$ of $Y$. We claim that $x \in \operatorname{patch}(Y)$. Suppose otherwise. Then there exists a quasicompact open subset $U$ of $X$, and a subset $V$ of $X$ that is the complement of a quasicompact open subset of $X$, such 
that $Y \subseteq U \cup V$ but $x \notin U \cup V$. If $Z \cap U$ is nonempty, then for any $z$ in this intersection, $z \in Z \subseteq \operatorname{cl}(\{x\})$, so that since $z$ is in the open set $U$, so is $x$, a contradiction. Thus $Z \cap U$ is empty, and hence $Z \subseteq V$, so that $x \in \operatorname{cl}(Z) \subseteq V$, again a contradiction. Therefore, $x \in \operatorname{patch}(Y)$.

(2) Suppose that $Y$ is a retrocompact subspace of $X$. In light of (1), to prove (2) all that needs to be shown is that if $x \in \operatorname{patch}(Y)$, then $x$ is a generic point for some subset of $Y$. Let $x \in \operatorname{patch}(Y)$. Then $\operatorname{cl}(\operatorname{cl}(\{x\}) \cap Y) \subseteq \operatorname{cl}(\{x\})$. We claim that this set inclusion is an equality. By way of contradiction, suppose this inclusion is proper, and let $U=(Y \cup\{x\}) \backslash \operatorname{cl}(\{x\})$ and $V=\operatorname{cl}(\operatorname{cl}(\{x\}) \cap Y)$. Then $Y \subseteq U \cup V$ but $x \notin U \cup V$. Since the closed set $V$ is an intersection of complements of quasicompact open subsets of $X$ and $x \notin V$, there exists a set $V^{\prime}$ such that $x \notin V^{\prime}, V \subseteq V^{\prime}$ and $V^{\prime}$ is the complement of a quasicompact open subset of $X$. Also, since $Y$ is retrocompact, $Y \backslash V^{\prime}=Y \cap\left(X \backslash V^{\prime}\right)$ is quasicompact, so since $Y \backslash V^{\prime} \subseteq U$ and $U$ is a union of quasicompact open subsets of $X$, it follows that there exists a quasicompact open subset $U^{\prime}$ such that $Y \backslash V^{\prime} \subseteq U^{\prime} \subseteq U$. Now $Y \subseteq U^{\prime} \cup V^{\prime}$, and since $U^{\prime} \subseteq U$, then $x \notin U^{\prime}$, and by the choice of $V^{\prime}, x \notin V^{\prime}$. Thus $Y \subseteq U^{\prime} \cup V^{\prime}$ but $x \notin U^{\prime} \cup V^{\prime}$, which is impossible since $x \in \operatorname{patch}(Y)$. This shows that every element of $\operatorname{patch}(Y)$ is a generic point for some subset of $Y$.

We next apply these notions to projective limits of Noetherian spectral spaces. Theorem 2.9 is our main result in this direction, and it follows from two preliminary lemmas.

Lemma 2.7. Let $d: Y \rightarrow X$ be a spectral map between spectral spaces, and let $Z$ be a subspace of $Y$. Then:

(1) The mapping $d$ is continuous in the patch and inverse topologies.

(2) $d(\operatorname{gen}(Z)) \subseteq \operatorname{gen}(d(Z)), d(\operatorname{inv}(Z)) \subseteq \operatorname{inv}(d(Z))$ and $d(\operatorname{patch}(Z))=\operatorname{patch}(d(Z))$.

(3) If $d$ is a closed map, then $d(\operatorname{pt}(Z))=\operatorname{pt}(d(Z))$.

(4) Each point in $X$ that is a generic point for a subset of $d(Z)$ is in $d(\operatorname{patch}(Z))$.

(5) If also $X$ is a Noetherian spectral space, then $\operatorname{pt}(d(Z))$ is the set of points that are closed in the subspace $d(Z)$ of $X$.

Proof. (1) This follows from the relevant definitions.

(2) To see that $d(\operatorname{gen}(Z)) \subseteq \operatorname{gen}(d(Z))$, let $x \in \operatorname{gen}(Z)$. Then there exists $z \in Z$ such that $z \in \operatorname{cl}(\{x\})$. Hence $d(z) \in d(\operatorname{cl}(\{x\})) \subseteq \operatorname{cl}(\{d(x)\})$, so that $d(x) \in \operatorname{gen}(d(z))$. This shows that $d(\operatorname{gen}(Z)) \subseteq \operatorname{gen}(d(Z))$. Also, since $d$ is continuous in the inverse topology, it follows that $d(\operatorname{inv}(Z)) \subseteq \operatorname{inv}(d(Z))$. Finally, since the patch topology is compact and Hausdorff and a continuous map between compact Hausdorff spaces is closed, the mapping $d$ is closed in the patch topology, and hence $d(\operatorname{patch}(Z))=\operatorname{patch}(d(Z))$.

(3) Suppose that $d$ is a closed map. To show that $d(\operatorname{pt}(Z)) \subseteq \operatorname{pt}(d(Z))$, let $x \in d(\operatorname{pt}(Z))$. Then there exists $y \in \operatorname{pt}(Z)$ such that $d(y)=x$. By Proposition $2.1(2), \operatorname{pt}(Z) \subseteq \operatorname{patch}(Z)$, so $y$ is a closed point in patch $(Z)$. Since $d$ is a closed map and $y$ is a closed point in patch $(Z)$, then $d(y)$ is a closed in $d(\operatorname{patch}(Z))=\operatorname{patch}(d(Z))$. It follows then that $d(y)$ is a closed point in $\operatorname{inv}(d(Z))$, and hence $x \in \operatorname{pt}(d(Z))$.

Conversely, if $x \in \operatorname{pt}(d(Z))$, then $x$ is a closed point in $\operatorname{inv}(d(Z))$, and hence a closed point in patch $(d(Z))$. By Lemma $2.7(2), \operatorname{patch}(d(Z))=d(\operatorname{patch}(Z))$, so $x$ is a closed 
point in $d(\operatorname{patch}(z))$. As such, $d^{-1}(x) \cap \operatorname{patch}(Z)$ is a closed set in $\operatorname{patch}(Z)$. Since by Proposition 2.1(1), patch $(Z)$, and hence $d^{-1}(x) \cap \operatorname{patch}(Z)$, is a spectral space, there exists $y \in \operatorname{patch}(Z) \operatorname{such}$ that $y$ is a closed point in the closed subset $d^{-1}(x) \cap \operatorname{patch}(Z)$ of $\operatorname{patch}(Z)$. Thus $y$ is a closed point in $\operatorname{patch}(Z)$ with $d(y)=x$. Since by Proposition 2.1(3), $\operatorname{inv}(Z)=$ $\operatorname{gen}(\operatorname{patch}(Z))$, it follows that $y$ is a closed point in $\operatorname{inv}(Z)$, so that $y \in \operatorname{pt}(Z)$. Therefore, $x=d(y) \in d(\operatorname{pt}(Z))$, which proves that $\operatorname{pt}(d(Z))=d(\operatorname{pt}(Z))$.

(4) Suppose there exists a subset $Y$ of $Z$ such that $x \in X$ is a generic point for $d(Y)$. Then by Proposition 2.6(1), $x \in \operatorname{patch}(d(Y))$, and hence by (2), $x \in d(\operatorname{patch}(Y))$.

(5) Let $x$ be a closed point in $\operatorname{inv}(d(Z))$. Then since by assumption $X$ is a Noetherian spectral space, Proposition 2.2 implies that $x \in \operatorname{pt}(d(Z)) \subseteq d(Z)$. Thus $x$ is a closed point in $d(Z)$. Conversely, let $y$ be a closed point in $d(Z)$. Then $y \in \operatorname{inv}(d(Z))$, so by Proposition 2.1 since $\operatorname{inv}(d(Z))$ is a spectral space there exists a closed point $w \in \operatorname{inv}(d(Z))$ such that $w \in \operatorname{cl}(\{y\})$. The preceding argument shows that $w$ is a closed point in $d(Z)$. But $y$ is a closed point in $d(Z)$, so since $w \in \operatorname{cl}(\{y\}) \cap d(Z)$, it follows that $y=w$ and $y$ is a closed point in $\operatorname{inv}(d(Z)))$; i.e., $y \in \operatorname{pt}(d(Z))$.

The operators patch and inv also behave well with respect to projective limits.

Lemma 2.8. Let $Y$ be a spectral space that is a projective limit (in the category of spectral spaces with spectral maps) of a projective system $\mathfrak{S}$ of spectral spaces. Let $Z$ be a subset of $Y$. For each $X \in \mathfrak{S}$, let $d_{X}: Y \rightarrow X$ denote the projection, and let $X(Z)=d_{X}(Z)$. Then $\{\operatorname{patch}(X(Z)): X \in \mathfrak{S}\}$ and $\{\operatorname{inv}(X(Z)): X \in \mathfrak{S}\}$ form projective systems with the induced maps. As topological spaces,

$$
\operatorname{patch}(Z)=\lim _{\longleftarrow} \operatorname{patch}(X(Z)) \quad \text { and } \operatorname{inv}(Z)=\lim _{\longleftarrow} \operatorname{inv}(X(Z)),
$$

where in each case $X$ ranges over the spaces in $\mathfrak{S}$.

Proof. If $d: X_{1} \rightarrow X_{2}$ is a map in the projective system $\mathfrak{S}$, then Lemma 2.7 implies that $d\left(\operatorname{patch}\left(X_{1}(Z)\right)\right)=\operatorname{patch}\left(X_{2}(Z)\right)$ and $d\left(\operatorname{inv}\left(X_{1}(Z)\right)\right) \subseteq \operatorname{inv}\left(X_{2}(Z)\right)$. It follows that $\{\operatorname{patch}(X(Z)): X \in \mathfrak{S}\}$ and $\{\operatorname{inv}(X(Z)): X \in \mathfrak{S}\}$ form projective systems with the induced maps. Now patch $(-)$ is a closure operator in the patch topology and the projection mappings are continuous in the patch topology (Lemma2.7(1)), so patch $(Z)$ is the projective limit of the spectral spaces patch $(X(Z))$ [4, Corollary, p. 49]. Similarly, since inv(-) is a closure operator in the inverse topology and the projections mappings are continuous in the inverse topology (again by Lemma 2.7(1)), then $\operatorname{inv}(Z)$ is the projective limit of the $\operatorname{inv}(X(Z))$.

The lemmas lead to the main theorem of the section, a characterization of inv, patch and pt in the case where the spectral spaces in $\mathfrak{S}$ are Noetherian. This theorem will be applied to the Zariski-Riemann space in Corollary 3.4.

Theorem 2.9. With the assumptions of Lemma 2.8, suppose in addition that each space in $\mathfrak{S}$ is Noetherian. Then:

$$
\begin{aligned}
\operatorname{inv}(Z) & =\left\{y \in Y: \forall X \in \mathfrak{S}, d_{X}(y) \text { specializes to a point in } X(Z)\right\} \\
\operatorname{patch}(Z) & =\left\{y \in Y: \forall X \in \mathfrak{S}, d_{X}(y) \text { is a generic point of a subset of } X(Z)\right\}
\end{aligned}
$$


If also each $d_{X}$ is a closed map, then

$$
\operatorname{pt}(Z)=\left\{y \in Y: \forall X \in \mathfrak{S}, d_{X}(y) \text { is a closed point in } X(Z)\right\} .
$$

Proof. To prove the first assertion, let $y \in \operatorname{inv}(Z)$, and let $X \in \mathfrak{S}$. Then by Lemma $2.7(2)$, $d_{X}(y) \in X(\operatorname{inv}(Z)) \subseteq \operatorname{inv}(X(Z))$. Since $X$ is Noetherian, so is every subspace of $X$, and hence $X(Z)$ is quasicompact. Thus by Proposition 2.2 $d_{X}(y) \in X(\operatorname{inv}(Z)) \subseteq \operatorname{inv}(X(Z))=$ gen $(X(Z))$, and hence $d_{X}(y)$ specializes to a point in $X(Z)$. Conversely, suppose that $y \in Y$ has the property that for each $X \in \mathfrak{S}, d_{X}(y)$ specializes to a point in $X(Z)$. Then $d_{X}(y) \in \operatorname{gen}(X(Z))=\operatorname{inv}(X(Z))$ for each $X \in \mathfrak{S}$. Thus $y$ is in $\lim _{\longleftarrow} \operatorname{inv}(X(Z))$ [4 , Corollary, p. 49], so that by Lemma 2.8 $y \in \operatorname{inv}(Z)$.

Next, let $y \in \operatorname{patch}(Z)$, and let $X \in \mathfrak{S}$. By Lemma $2.7(2), d_{X}(y) \in X(\operatorname{patch}(Z))=$ $\operatorname{patch}(X(Z))$, and hence by Proposition 2.6, $d_{X}(y)$ is a generic point for a subset of $X(Z)$. Conversely, let $y \in Y$ and suppose that for all $X \in \mathfrak{S}, d_{X}(y)$ is a generic point of a subset of $X(Z)$. Then by Proposition 2.6 $d_{X}(y) \in \operatorname{patch}(X(Z))$. As in the case of $\operatorname{inv}(Z)$, this implies that $y \in \operatorname{patch}(Z)$.

To verify the assertion about $\operatorname{pt}(Z)$, let $y \in \operatorname{pt}(Z)$, and let $X \in \mathfrak{S}$. Then by Proposition 2.2 and Lemma 2.7(3), $d_{X}(y) \in X(\operatorname{pt}(Z))=\operatorname{pt}(X(Z)) \subseteq X(Z)$. Hence $d_{X}(y)$ is a closed point in $X(Z)$. Conversely, suppose that $y \in Y$ and for each $X \in \mathfrak{S}, d_{X}(y)$ is a closed point of $X(Z)$. Since $X(Z)$ is quasicompact, $\operatorname{gen}(X(Z))=\operatorname{inv}(X(Z))$, and hence $d_{X}(y)$ is a closed point in $\operatorname{inv}(X(Z))$. Since by Lemma 2.8, $\operatorname{inv}(Z)=\lim \operatorname{inv}(X(Z))$, then it follows that $y$ is a closed point in $\operatorname{inv}(Z)$, and hence $y \in \operatorname{pt}(Z)$.

\section{The Zariski-Riemann space as a limit of projective models}

A projective model $X$ of $F / D$ is a projective integral scheme over $\operatorname{Spec}(D)$ whose function field is a subfield of $F$. Because we wish for our framework to be flexible enough to handle the case in which $D$ is the prime subring of $F$, we depart from contemporary usage and follow Zariski-Samuel [45, Chapter VI, §17] in that we do not require a projective model of $F / D$ to have function field $F$. However, using the notion of a dominant system of projective models mentioned below, we can reduce to this case when it is possible; that is, when $F$ is a finitely generated field extension of the quotient field of $D$.

Each projective model $X$ can be realized in a convenient way. Namely, there exist $f_{1}, \ldots, f_{n} \in F$ such that if for each $i, D_{i}=D\left[\frac{f_{1}}{f_{i}}, \ldots, \frac{f_{n}}{f_{i}}\right]$, then $X=\bigcup_{i} \operatorname{Spec}\left(D_{i}\right)$, where prime ideals $\mathfrak{p}_{i}$ of $D_{i}$ and $\mathfrak{p}_{j}$ of $D_{j}$ are identified in $X$ whenever $\left(D_{i}\right)_{\mathfrak{p}_{i}}=\left(D_{j}\right)_{\mathfrak{p}_{j}}$. We denote the structure sheaf of $X$ by $\mathcal{O}_{X}$. For each nonempty open subset $U$ of $X$, we view the stalk $\mathcal{O}_{X, x}$ as a subring of $F$, so that $\mathcal{O}_{X}(U)=\bigcap_{x \in U} \mathcal{O}_{X, x}$. The Zariski topology on $X$ has as a basis of open sets the sets of the form $\left\{x \in X: f_{1}, \ldots, f_{n} \in \mathcal{O}_{X, x}\right\}$, where $f_{1}, \ldots, f_{n} \in F$.

A projective model $Y$ of $F / D$ dominates a projective model $X$ of $F / D$ if for each $y \in Y$, there exists $x \in X$ such that $\mathcal{O}_{X, x} \subseteq \mathcal{O}_{Y, y}$ and $\mathfrak{m}_{X, x}=\mathfrak{m}_{Y, y} \cap \mathcal{O}_{X, x}$. Thus when $Y$ dominates $X$, there is a domination morphism $\delta_{X}^{Y}=\left(d, d^{\#}\right): Y \rightarrow X$, where the map $d: Y \rightarrow X$ on the underlying topological spaces is given by $d(y)=x$ (with $x$ as above) and the sheaf morphism $d^{\#}: \mathcal{O}_{X} \rightarrow d_{*} \mathcal{O}_{Y}$ is defined on each nonempty open subset $U$ of $X$ by $d^{\#}(U): \mathcal{O}_{X}(U) \rightarrow \mathcal{O}_{Y}\left(d^{-1}(U)\right): s \mapsto s$. The mapping $d$ is continuous and closed [45, Lemma 5, p. 119], and $\delta_{X}^{Y}$ is a dominant morphism of schemes. 
Given any two projective models $X$ and $Y$ of $F / D$, there exists a projective model $Z$ of $F / D$ (namely, $Z=X \times \times_{\operatorname{Spec}(D)} Y$ ) such that $Z$ dominates both $X$ and $Y$ [45, Lemma 6 , p. 119], and hence the set of projective models of $F / D$ equipped with the domination morphisms forms a projective system, a fact we revisit in Proposition 3.3 . Similarly, $\mathfrak{X}$ dominates each projective model $X$ of $F / D$ in the sense that for each $x \in X$, there exists a valuation ring $V \in \mathfrak{X}$ such that $\mathcal{O}_{X, x} \subseteq V$ and $\mathfrak{m}_{X, x}=\mathfrak{M}_{V} \cap \mathcal{O}_{X, x}$, where $\mathfrak{M}_{V}$ is the maximal ideal of $V$ [45, pp. 119-120]. We say that $V$ is centered on $x$. In fact, there is a morphim of locally ringed spaces $\delta_{X}^{\mathfrak{X}}=\left(d, d^{\#}\right): \mathfrak{X} \rightarrow X$, where $d(V)=x$ (with $x$ as above), and $d^{\#}: \mathcal{O}_{X} \rightarrow d_{*} \mathcal{O}_{\mathfrak{X}}$ is defined on nonempty open subsets $U$ of $X$ by $d_{I J}^{\#}: \mathcal{O}_{X}(U) \rightarrow \mathcal{O}_{\mathfrak{X}}\left(d^{-1}(U)\right): s \mapsto s$. The mapping $d$ is surjective, continuous and closed [45, Lemma 4, p. 117]. Abstractly, the domination morphism $\delta_{X}^{\mathfrak{X}}$ encodes the valuative criteria for properness [21, Theorem II.4.7].

For lack of a reference, we note next that the underlying topological space of a projective model is a spectral space. The proof of the proposition uses the fact that $\mathfrak{X}$ is a spectral space, which we discuss more in Remark 3.2 and the next section.

Proposition 3.1. The underlying topological spaces of a projective model $X$ of $F / D$ is a spectral space. If also $\operatorname{Spec}(D)$ is a Noetherian space, then $X$ is a Noetherian spectral space.

Proof. Let $X$ be a projective model of $F / D$. Then $X$ is a $T_{0}$-space having a basis of quasicompact open subsets. Moreover, as discussed above, the domination mapping $d$ : $\mathfrak{X} \rightarrow X$ of the underlying topological spaces is a continuous surjective mapping, and, as noted below in Remark 3.2 $\mathfrak{X}$ is a spectral space. Thus by [10, Proposition 9], to prove that $X$ is a spectral space, it suffices to show that $d^{-1}(U)$ is a quasicompact subset of $\mathfrak{X}$ for every quasicompact open subset $U$ of $X$. Let $U$ be a basic open subset of $X$. Then there exist $f_{1}, \ldots, f_{n} \in F$ such that $U=\left\{x \in X: f_{1}, \ldots, f_{n} \in \mathcal{O}_{X, x}\right\}$, and hence $d^{-1}(U)=\{V \in \mathfrak{X}$ : $\left.f_{1}, \ldots, f_{n} \in V\right\}$ is a quasicompact open subset of $\mathfrak{X}$. More generally, if $U$ is a quasicompact open subset of $X$, then $U=U_{1} \cup \cdots \cup U_{m}$ for basic open subsets $U_{i}$ of $X$, and it follows that $d^{-1}(U)$ is a quasicompact subset of $\mathfrak{X}$. Therefore, $X$ is a spectral space. Suppose finally that $\operatorname{Spec}(D)$ is a Noetherian space. The projective model $X$ of $F / D$ is covered by finitely many affine submodels $\operatorname{Spec}\left(D_{i}\right), i=1, \ldots, m$, where each $D_{i}$ is a finitely generated $D$-subalgebra of $F$. A finitely generated algebra over a ring with Noetherian prime spectrum again has Noetherian prime spectrum [31, Corollary 2.6], and so for each $i, \operatorname{Spec}\left(D_{i}\right)$ is a Noetherian space. It follows that $X$, as a finite union of Noetherian subspaces, is a Noetherian space.

Remark 3.2. Viewing $\mathfrak{X}$ and $X$ as spectral spaces, the proof of the proposition shows that the map $d: \mathfrak{X} \rightarrow X$ is a spectral map. That $d$ is a spectral map was first observed by Dobbs, Fedder and Fontana, who gave a topological proof that when $X=\operatorname{Spec}(D)$ and $D$ has quotient field $F$, then $\mathfrak{X}$ is a spectral space and $d$ is a spectral map [9, Theorem 4.1]. Dobbs and Fontana presented a sharper version of this result in [10, Theorem 2] by exhibiting a ring $R$ (namely, the Kronecker function ring of $R$ with respect to the $b$-operation, which is discussed in the next section) such that $\mathfrak{X}$ is homeomorphic to $\operatorname{Spec}(R)$. That the ZariskiRiemann space $\mathfrak{X}$ of $F / D$ is a spectral space when $D$ does not necessarily have quotient field $F$ follows from [23, Proposition 2.7] or [16, Corollary 3.6]. In the appendix of [28], Kuhlmann gives a model-theoretic proof of the fact that $\mathfrak{X}$ is a spectral space.

When $X$ is a projective model of $F / D$ and $\operatorname{Spec}(D)$ is a Noetherian space, then by Proposition 3.1, $X$ is a Noetherian space, so that by Corollary 2.3, $\operatorname{inv}(Y)=\operatorname{gen}(Y)$ for 
every subset $Y$ of $X$. By contrast, subspaces of $\mathfrak{X}$ are generally not quasicompact, and the inverse topology is more nuanced for this spectral space, as we see throughout the rest of the article. We will see also that the patch topology is more subtle on $\mathfrak{X}$ than on the projective model $X$, the latter having been described in Proposition 2.6.

We say a collection $\mathfrak{S}$ of projective models of $F / D$ is a dominant system if for each pair of projective models $X$ and $Y$ of $F / D$ there exists a projective model $Z \in \mathfrak{S}$ that dominates both $X$ and $Y$. The set of all projective models of $F / D$ is a dominant system 445, Lemma 6 , p. 120]. On the other hand, if $F$ is finitely generated over the quotient field of $D$, then the set of projective models of $F / D$ having function field $F$ is also a dominant system. In this second case, the notion of a dominant system gives us a convenient way to restrict to the birational setting.

Let $\mathfrak{S}$ be a dominant system of projective models. Since each pair of projective models in $\mathfrak{S}$ is dominated by another in $\mathfrak{S}$, then $\mathfrak{S}$ forms a projective system in the category of locally ringed spaces. Moreover, for each $X \in \mathfrak{S}$, we have the domination morphism $\delta_{X}^{\mathfrak{X}}: \mathfrak{X} \rightarrow X$. While not stated in this terminology, most of the following proposition is implicit in Zariski-Samuel [45, Theorem VI.41, p. 122].

Proposition 3.3. (Zariski-Samuel) Let $\mathfrak{S}$ be a dominant system of projective models of $F / D$.

(1) As a topological space, $\mathfrak{X}$ is the projective limit in the category of topological spaces of the underlying topological spaces of the projective models in $\mathfrak{S}$.

(2) As a spectral space, $\mathfrak{X}$ is the projective limit in the category of spectral spaces of the underlying spectral spaces of the projective models in $\mathfrak{S}$. Moreover, for each $X \in \mathfrak{S}$, the map of spectral spaces underlying the domination morphism $\delta_{X}^{\mathfrak{X}}$ is a closed spectral map.

(3) Each $V \in \mathfrak{X}$ is the union of the $\mathcal{O}_{X, x_{V}}$, where $X$ ranges over $\mathfrak{S}$ and $x_{V}$ is the center of $V$ in $X$.

(4) As locally ringed spaces, $\mathfrak{X}$ is the projective limit of the projective models in $\mathfrak{S}$.

Proof. Statements (1) and (3) follow from the proof of Theorem VI.41, p. 122, in [45]. Details for the proof of statement (4) can be found in [27, Theorem 2.1.5]. To see that (2) holds, note that the domination morphisms $\delta_{X}^{Y}: Y \rightarrow X$, where $Y$ and $X$ are projective models in $\mathfrak{S}$ and $Y$ dominates $X$, are of finite type, and hence are quasicompact 21, Exercise II.3.2, p. 91]. Thus the underlying continuous maps of the domination morphisms between projective models in $\mathfrak{S}$ are spectral maps. It follows then from (1) that $\mathfrak{X}$ is the projective limit in the category of spectral spaces of the underlying spectral spaces of the projective models in $\mathfrak{S}$. That the underlying map of the domination morphism $\delta_{X}^{\mathfrak{X}}$ is a closed spectral map was discussed above.

When $X$ is a projective model of $F / D$ and $Z$ is a subset of $\mathfrak{X}$ we set

$$
X(Z)=\text { the image of } Z \text { in } X \text { under the domination morphism } \delta_{X}^{\mathfrak{X}} \text {. }
$$

Thus a point $x \in X$ is in $X(Z)$ if and only if there is a valuation ring $V$ in $Z$ centered on $x$. 
Corollary 3.4. Suppose $\operatorname{Spec}(D)$ is a Noetherian space. Let $\mathfrak{S}$ be a dominant system of projective models of $F / D$ and let $Z$ be a subspace of $\mathfrak{X}$. Then:

$$
\begin{aligned}
\operatorname{inv}(Z) & =\{V \in \mathfrak{X}: \forall X \in \mathfrak{S}, \text { the center of } V \text { in } X \text { specializes to a point in } X(Z)\} \\
\operatorname{patch}(Z) & =\{V \in \mathfrak{X}: \forall X \in \mathfrak{S}, V \text { is centered on a generic point of a subset of } X(Z)\} \\
\operatorname{pt}(Z) & =\{V \in \mathfrak{X}: \forall X \in \mathfrak{S}, V \text { is centered on a closed point in } X(Z)\} .
\end{aligned}
$$

Proof. Since $\operatorname{Spec}(D)$ is a Noetherian space, each projective model of $F / D$ is by Proposition 3.1 a Noetherian spectral space. Thus we may apply Theorem[2.9 and Proposition 3.3(3) to obtain the corollary.

An example shows that without the assumption in the theorem that $\operatorname{Spec}(D)$ is a Noetherian space, the characterization of $\operatorname{inv}(Z)$ need not be valid.

Example 3.5. Let $R$ be a Prüfer domain with quotient field $F$, and suppose that $R$ has a maximal ideal $M$ such that $\bigcap_{N \neq M} R_{N} \subseteq R_{M}$, where $N$ ranges over the maximal ideals of $R$ distinct from $M$. Examples of such Prüfer domains include the ring of entire functions [17, Proposition 8.1.1(6), p. 276], holomorphy and Kronecker function rings of function fields of transcendence degree $>1$ ([32, Theorem 4.7] and 23, Theorem 4.3], respectively), and the ring of integer-valued polynomials (e.g., combine [19, Theorem 1.6] and the proof of Proposition VI.2.8 in [7].) Then $X:=\operatorname{Spec}(R)$ is a projective model of $F / R$. Set $Z_{2}=\left\{R_{N}: N \in \operatorname{Max}(R) \backslash\{M\}\right\}$ and $Z_{1}=\operatorname{Max}(R)$. Then $\bigcap_{V \in Z_{1}} V=\bigcap_{V \in Z_{2}} V$, and since the fact that $R$ is a Prüfer domain implies that $\mathfrak{X}$ is an affine scheme (see Section 4 ), then $\operatorname{inv}\left(Z_{1}\right)=\operatorname{inv}\left(Z_{2}\right)$ (see Proposition 5.6(5)). Thus $R_{M} \in \operatorname{inv}\left(Z_{1}\right)=\operatorname{inv}\left(Z_{2}\right)$, but the closure of the center $M$ of the valuation ring $R_{M} \in Z_{1}$ in $X$ is simply $\{M\}$, which does not meet $X\left(Z_{2}\right)$, in contrast to the characterization in Corollary 2.9.

Remark 3.6. Suppose $D$ is a field and $F$ is a finitely generated field extension of $D$. Let $Z \subseteq \mathfrak{X}$. Favre has shown that for any valuation ring $V \in \operatorname{cl}(Z)$, either $V \in \operatorname{cl}(\{U\})$ for some $U \in Z$ or there is a sequence $\left\{V_{i}\right\}_{i=1}^{\infty}$ of valuation rings in $Z$ such that $V$ is the limit in the Zariski topology on $\mathfrak{X}$ of the $V_{i}[14$, Theorem 3.1]. Thus every valuation ring in $\operatorname{cl}(Z) \cap \operatorname{gen}(Z)$ is a limit of a (countable) sequence of valuation rings in $Z$. It follows also from [14, Lemma 2.4] that in the terminology of Remark [3.9(1), every valuation ring in $\operatorname{cl}(Z) \cap \operatorname{gen}(Z)$ is an ultrafilter limit of countably many valuation rings in $Z$.

In Corollary 3.8 we prove patch density of some canonically chosen collections of valuation rings. The corollary is a consequence of a fact about the "degenerate" case for the intersection of valuation rings in a subspace of $\mathfrak{X}$. Recall that a projective model $X$ having function field $F$ is normal if $X$ is defined by $f_{0}, \ldots, f_{n} \in F$ such that the rings $D_{i}=D\left[\frac{f_{0}}{f_{i}}, \ldots, \frac{f_{n}}{f_{i}}\right]$ in the resulting open cover $X=\bigcup_{i=0}^{n} \operatorname{Spec}\left(D_{i}\right)$ are all integrally closed in $F$.

Proposition 3.7. Suppose $D$ is a finitely generated algebra over a field. If $X$ is a normal projective model of $F / D$ having function field $F$, and $Z$ is a subset of $\mathfrak{X}$ such that $X(Z)$ contains all but at most finitely many closed points of $X$, then $A=\bigcap_{V \in Z} V$ is the integral closure of $D$ in $F$. 
Proof. There exist $f_{1}, \ldots, f_{n} \in F$ such that with $D_{i}=D\left[\frac{f_{1}}{f_{i}}, \ldots, \frac{f_{n}}{f_{i}}\right]$, then each $D_{i}$ is an integrally closed Noetherian domain with quotient field $F$ and $X=\bigcup_{i} \operatorname{Spec}\left(D_{i}\right)$. Fix $i$, and let $\mathfrak{p}$ be a height 1 prime ideal of $D_{i}$. Then since $D_{i}$ is a Hilbert domain, $\mathfrak{p}$ is an intersection of infinitely many maximal ideals of $D_{i}$. Now since all but at most finitely many maximal ideals of $D_{i}$ are in $X(Z)$, it follows that the point in $X$ corresponding to $\mathfrak{p}$ is a generic point of a subset of $X$. Therefore, by Proposition 2.6 $\mathfrak{p}$ is in $\operatorname{patch}(X(Z))$. Since the domination map $Z \rightarrow X$ is a closed spectral map, Lemma 2.7(4) implies that each point in $X$ that is a generic point for a subset of $X(Z)$ has a valuation ring in $\operatorname{patch}(Z)$ centered on it. Thus since $\left(D_{i}\right)_{\mathfrak{p}}$ is a DVR, it must be that $\left(D_{i}\right)_{\mathfrak{p}} \in \operatorname{patch}(Z) \subseteq \operatorname{inv}(Z)$. This is true for every choice of $i$ and height 1 prime ideal $\mathfrak{p}$ of $D_{i}$, so since each $D_{i}$ is a Krull domain, we conclude that $A \subseteq D_{1} \cap \cdots \cap D_{n}$. Moreover, every valuation ring in $\mathfrak{X}$ contains at least one of the rings $D_{i}$. Thus $A$ is the intersection of all valuation rings in $\mathfrak{X}$, so that $A$ is the integral closure of $D$ in $F$.

Thus with the assumptions on $D$, if for each closed point $x$ in $X$, the fiber $Z_{x}$ of the domination mapping $\delta: Z \rightarrow X$ is nonempty, then $A$ is the integral closure of $D$ in $F$. We use this observation to prove from a different point of view a density result due to Kuhlmann. His result is stronger than what is stated here, and is a consequence of a powerful existence theorem for valuations on function fields.

Corollary 3.8. (Kuhlmann 28, Theorem 9]) Let $D$ be a subfield of $F$, and suppose that $F / D$ is a finitely generated field extension of transcendence $n \geq 1$. Let $d \geq 0$ and $r \geq 1$ such that $n \geq d+r$, and let $Z_{d, r}$ be the set of all discrete valuation rings of rank $r$ whose residue field is a finitely generated field extension of $D$ having transcendence degree $d$. Then $Z_{d, r}$ is patch dense in $\mathfrak{X}$.

Proof. Let $X$ be a projective model of $F / D$. Then since $X=\bigcup_{i=1}^{n} \operatorname{Spec}\left(D_{i}\right)$, where each $D_{i}$ is a Hilbert domain, it follows as in the proof of Proposition 3.7 that the patch closure of the set of Zariski closed points of $X$ is $X$. Thus in light of Lemma 2.8 it suffices to show that when $X$ is a projective model of $F / D$ with function field $F(X)$, then every closed point in $X$ has a valuation ring in $Z_{d, r}$ centered on it. This is a standard fact about the valuation theory of function fields; for example, it follows from [3, Chapter VI, $\S 10.3$, Theorem 1] (but see the version stated in [29, Lemma 2.6]).

As a particular case of the corollary, the set $Z$ of discrete rank one valuation rings (DVRs) $V$ in $\mathfrak{X}$ whose residue fields are finite over $k$ is patch dense in $\mathfrak{X}$. This has the interesting consequence that every valuation ring in $\mathfrak{X}$ is an ultrafilter limit of valuation rings in $Z$; see Remark 3.9(1) below. These DVRs in $Z$ are particularly tractable, since they arise from prime ideals in the generic formal fiber of local rings of closed points in projective models of $F / k$; see $[22,2.6$, p. 25].

In the context of the corollary, when $k$ is a perfect field, then the set $Z$ of DVRs in $\mathfrak{X}$ having residue field $k$ lie dense with respect to the patch topology in the space $Z^{\prime}$ of all valuation rings in $\mathfrak{X}$ having residue field $k$ [28, Corollary 5]. If also $k$ is not algebraically closed, then as noted in Example 4.1 $Z$, and hence $Z^{\prime}$, are "affine" sets, as defined in the next section.

\section{Remark 3.9.}


(1) The patch closure in $\mathfrak{X}$ has a helpful interpretation in terms of ultrafilter limits of valuation rings. This is developed in [16, Corollary 3.8], where it is shown that when $Z$ is a subset of $\mathfrak{X}$, then $\operatorname{patch}(Z)$ is the set of valuation rings $V$ in $\mathfrak{X}$ of the form

$$
V=\{x \in F:\{V \in Z: x \in V\} \in \mathcal{U}\},
$$

where $\mathcal{U}$ is an ultrafilter on the set $Z$.

(2) A version of the Zariski-Riemann space has been developed for graded valuation rings by Temkin in [42], where it is shown that the graded Zariski-Riemann space is quasicompact [42, Lemma 2.1]. In [11, Theorem 1.5], Ducros shows that certain maps between graded Zariski-Riemann spaces are closed with respect to the inverse topology. The patch topology on the graded Zariski-Riemann space is considered by Conrad and Temkin in [8, Lemma 5.3.6].

(3) With $D=\mathbb{C}[[X, Y]], F=\mathbb{C}((X, Y))$ and $\mathfrak{X}_{0}$ the set of valuation rings in $F / D$ centered on the maximal ideal of $D$, Favre and Jonsson use the patch topology (the "HausdorffZariski" topology) on $\mathfrak{X}_{0}$ to describe features of a tree structure on $\mathfrak{X}_{0}$ whose partial ordering is determined by behavior with respect to sequences of blow-ups $[15$, Proposition 5.29, p. 107].

(4) Motivated by applications in real algebraic geometry and rigid analytic geometry, Huber introduced the valuation spectra of a ring as a generalization of the ZariskiRiemann surface of an extension $F / D$. The patch topology is often the topology of choice for valuation spectra; see Huber-Knebusch [25].

\section{The Zariski-Riemann space as the image of an affine scheme}

Again we assume $D$ is a subring of a field $F$ but we do not assume any additional conditions (e.g., Noetherian) on $D$. By Proposition 3.3, the locally ringed space $\mathfrak{X}$ is a projective limit of projective schemes, but is itself in general not a scheme. However, by using the Kronecker function ring construction from multiplicative ideal theory, we can view $\mathfrak{X}$ as the image of an affine scheme. We discuss how to do this in this section.

Let $T$ be an indeterminate for $F$. For each valuation $\operatorname{ring} V \in \mathfrak{X}$, let $V^{*}$ be the Gaussian extension of $V$ to $F(T)$ (= field of rational functions in the variable $T$ ); that is,

$$
V^{*}=V[T]_{\mathfrak{M}_{V}[T]} .
$$

Then $V^{*}$ is a valuation ring with quotient field $F(T)$ such that $V=V^{*} \cap F$. For a subset $Z$ of $\mathfrak{X}$, we define the Kronecker function ring of $Z$ to be the ring

$$
\operatorname{Kr}(Z)=\bigcap_{V \in Z} V^{*}
$$

In the special case in which $D$ has quotient field $F$, then $\operatorname{Kr}(\mathfrak{X})$ is the classical Kronecker function ring of $D$ with respect to the $b$-operation (see [18, Section 26]):

$$
\operatorname{Kr}(\mathfrak{X})=\left\{\frac{f}{g}: f, g \in D[T], g \neq 0 \text { and } \overline{c(f)} \subseteq \overline{c(g)}\right\},
$$


where $\bar{I}$ denote the integral closure of the ideal $I$ in $D$ (which in the notation of Krull is $I^{b}$, hence the terminology of " $b$-operation"). Although we will not need it, this classical description of the Kronecker function ring of $\mathfrak{X}$ with respect to $b$ can also be generalized to projective space [13].

In any case, when $Z$ is a subspace of $\mathfrak{X}$, then it follows from work of Halter-Koch on function rings that $\operatorname{Kr}(Z)$ is a Prüfer domain with quotient field $F(T)$; cf. [16, Corollary 3.6], 20, Theorem 2.2] and [23, Corollary 2.2]. Recall that a domain $R$ is a Prüfer domain if every localization of $R$ at a prime ideal is a valuation ring; equivalently, every valuation ring between $R$ and its quotient field is a localization of $R$ at a prime ideal. It follows then that if $R$ is a Prüfer domain containing $D$ and having quotient field $F$, then $\mathfrak{X}_{R} \rightarrow \operatorname{Spec}(R)$ is an isomorphism of locally ringed spaces, and hence $\mathfrak{X}_{R}$ has the structure of an affine scheme. Thus we obtain: For each subset $Z$ of $\mathfrak{X}$, the subspace $\mathfrak{X}_{\operatorname{Kr}(Z)}$ of $\mathfrak{X}^{*}:=\left\{V^{*}: V \in \mathfrak{X}\right\}$ is an affine scheme.

We are interested also in when a subspace of $\mathfrak{X}$ has the structure of an affine scheme. We characterize these subspaces in Theorem 6.1 as the inverse closed subspaces $Z$ of $\mathfrak{X}$ such that $A=\bigcap_{V \in Z} V$ is a Prüfer domain with quotient field $F$. Motivated by this characterization, we say a subset $Z$ of $\mathfrak{X}$ is affine if $A=\bigcap_{V \in Z} V$ is a Prüfer domain having quotient field $F$. This is a slight abuse of notation, since the possibly larger set $\operatorname{inv}(Z)$, but not $Z$ itself, is an affine scheme (in general, the affine set $Z$ is only a subset of an affine scheme). Thus an affine set is inverse dense in an affine scheme.

By the above remarks, there is a canonical way to associate to each $Z \subseteq \mathfrak{X}$ an affine set. Define

$$
Z^{*}=\left\{V^{*}: V \in Z\right\} .
$$

Then, since $\operatorname{Kr}(Z)=\bigcap_{V \in Z^{*}} V$, it follows that $Z^{*}$ is an affine subset of $\mathfrak{X}^{*}=\left\{V^{*}: V \in \mathfrak{X}\right\}$. In Section [5 we use this observation to describe subsets of $\mathfrak{X}$ that are closed in the inverse topology. By way of motivation, we mention here some examples of affine subsets of $\mathfrak{X}$.

\section{Example 4.1.}

(1) Any finite subset of $\mathfrak{X}$ is affine [30, (11.11), p. 38].

(2) If $D$ contains a field and $Z \subseteq \mathfrak{X}$ has cardinality less than the cardinality of this field, then $A=\bigcap_{V \in Z} V$ is a Prüfer domain, so that if also $A$ has quotient field $F$, then $Z$ is affine [35, Theorem 6.6].

(3) If $Z \subseteq \mathfrak{X}$ has the property that each valuation ring in $Z$ has a formally real residue field (i.e., -1 is not a sum of squares in the residue field), then $Z$ is an affine set. (The ring $A=\bigcap_{V \in Z} V$ is known in the literature of real algebraic geometry as the real holomorphy ring of $F / D$; see for example, [1, 2, 6, [5, 39].) This is a special case of a more general phenomenon: If there exists a nonconstant monic polynomial in $D[T]$ having no root in a residue field of any $V \in Z$, then $Z$ is affine. This result has been proved independently and in various forms by several authors, including A. Dress, R. Gilmer, K. A. Loper and P. Roquette; see [32, p. 332] for precise references and a discussion of this result.

(4) If $D$ contains a field $k$ that is not algebraically closed and every valuation ring in $Z \subseteq \mathfrak{X}$ has residue field $k$, then $Z$ is affine. This is a special case of the general result in (3). 
Returning to the case where $Z=\mathfrak{X}$, we have (see [23, Theorem 2.3]),

$$
\left(\mathfrak{X}^{*}\right)_{\operatorname{Kr}(\mathfrak{X})}=\mathfrak{X}^{*} .
$$

Thus since $\operatorname{Kr}(\mathfrak{X})$ is a Prüfer domain, the stalks on $\operatorname{Spec}(\operatorname{Kr}(\mathfrak{X}))$ are precisely the valuation rings in $\mathfrak{X}^{*}$. In this way, we obtain a morphism of locally ringed spaces $\kappa=\left(k, k^{\#}\right): \mathfrak{X}^{*} \rightarrow \mathfrak{X}$ defined in the following way. The map $k: \mathfrak{X}^{*} \rightarrow \mathfrak{X}$ is defined by $k(V)=V \cap F$ for each $V \in \mathfrak{X}^{*}$, and the morphism of sheaves $k^{\#}: \mathcal{O}_{\mathfrak{X}} \rightarrow k_{*} \mathcal{O}_{\mathfrak{X}^{*}}$ is defined for each nonempty open subset $U$ of $\mathfrak{X}$ by

$$
k_{U}^{\#}: \mathcal{O}_{\mathfrak{X}}(U) \rightarrow \mathcal{O}_{\mathfrak{X}^{*}}\left(k^{-1}(U)\right): s \mapsto s
$$

for each $s \in \mathcal{O}_{\mathfrak{X}}(U)$. The map $k$ is a homeomorphism; see [10] for the affine case and [16, Corollary 3.6] or [23, Proposition 2.7] for the general case.

This accomplishes our first goal of mirroring $\mathfrak{X}$ with the affine scheme $\operatorname{Spec}(\operatorname{Kr}(\mathfrak{X}))$, which in essence contains all of the valuation theory of $F / D$. Although this affine scheme is not birationally equivalent to the object $\mathfrak{X}$ (for it has function field $F(T)$ rather than $F$ ), we can change bases for each projective model $X$ of $F / D$ to produce a projective model $X^{*}$ of the extension $F(T) / D[T]$. This is done via the Nagata function ring,

$$
D^{*}:=\left\{\frac{f}{g}: f, g \in D[T], g \neq 0 \text { and } c(g)=D\right\},
$$

where $c(g)$ denotes the content of the polynomial $g$. (The ring $D^{*}$ is often denoted $D(T)$, but to be consistent with our other notation, we use $D^{*}$ rather than $D(T)$.) We let also $F^{*}=F(T)$. The ring $D^{*}$ is a faithfully flat extension of $D$. Therefore, faithfully flat base extension produces the desired projective model:

$$
X^{*}=X \times_{\operatorname{Spec}(D)} \operatorname{Spec}\left(D^{*}\right) .
$$

Thus when $X=\bigcup_{i=1}^{n} \operatorname{Spec}\left(D_{i}\right)$, we obtain that $X^{*}$ can be identified with $\bigcup_{i=1}^{n} \operatorname{Spec}\left(D^{*}\left[D_{i}\right]\right)$, and hence $X^{*}$ is a projective model of $F^{*} / D^{*}$. In the next proposition, we collect many of the preceding observations into a single diagram.

Proposition 4.2. With $X$ a projective model of $F / D$, there is a commutative diagram,

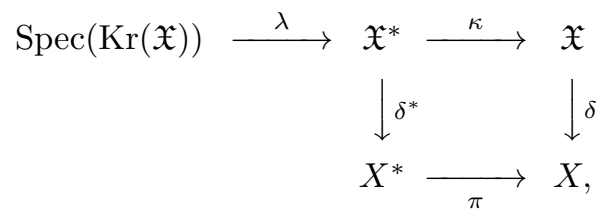

where:

(a) $\lambda$ is the isomorphism of locally ringed spaces induced by localization: $P \mapsto \operatorname{Kr}(\mathfrak{X})_{P}$ for each $P \in \operatorname{Spec}(\operatorname{Kr}(\mathfrak{X}))$;

(b) $\kappa: \mathfrak{X}^{*} \rightarrow \mathfrak{X}$ (which is defined above) is a morphism of locally ringed spaces that is a homeomorphism on the underlying topological spaces;

(c) $\pi$ is the surjective morphism of schemes given by faithfully flat base extension; 
(d) $\delta$ is the domination morphism and hence is a closed surjective morphism of locally ringed spaces; and

(e) $\delta^{*}$ is the morphism of locally ringed spaces given by the restriction to $\mathfrak{X}^{*}$ of the domination morphism from the Zariski-Riemann space of $F^{*} / D^{*}$ to $X^{*}$.

Each induced continous map in (a)-(e) on the underlying topological spaces is a spectral map, and hence is continuous in the Zariski, inverse and patch topologies.

Proof. Statements (a) and (b) were discussed above, and statement (c) is clear from the construction of $X^{*}$. Statement (d) was discussed in Section 3, and statement (e) follows similarly. The commutativity of the diagram is clear in light of the representation $X^{*}=$ $\bigcup_{i=1}^{n} \operatorname{Spec}\left(D^{*}\left[D_{i}\right]\right)$. To see that all the maps are spectral maps, observe that since the mappings $\lambda$ and $\kappa$ are homeomorphisms in the Zariski topology, it is clear that they are homeomorphisms in the inverse and patch topologies. By Remark 3.2 the continuous maps induced by $\delta$ and $\delta^{*}$ are spectral. Moreover, it is clear that $\pi$ is continuous, and since $\pi$ is a finite type, hence quasicompact, morphism, it induces a spectral map on the underlying topological spaces. Thus by Lemma 2.7(1), this map is continuous in the inverse and patch topologies.

Remark 4.3. The top row of the diagram in the proposition is also emphasized in 16 , Corollary 3.6 and Proposition 3.9]. That $\delta$ is continuous in the patch topology is proved in [16, Proposition 3.9] using an interpretation of patch closure involving ultrafilters (discussed in Remark [3.9). The argument there makes precise how $\delta$ preserves ultrafilter limits of valuation rings.

By the proposition, for each subset $Z$ of $\mathfrak{X}$, the subspace $Z^{*}$ of $\mathfrak{X}^{*}$ is homeomorphic to $Z$ via the restriction of $\kappa$ to $Z^{*}$. We rely on this observation in the next section, since it allows us when considering topological properties of $Z$ to replace a non-affine subset of $\mathfrak{X}$ with an affine subset of $\mathfrak{X}^{*}$ homeomorphic to $Z$.

Corollary 4.4. Let $X$ be a projective model of $F / D$, and let $Z$ be a subspace of $\mathfrak{X}$. Then the continuous map $X^{*} \rightarrow X$ induced by the morphism $\pi: X^{*} \rightarrow X$ restricts to a homeomorphism $X^{*}\left(Z^{*}\right) \rightarrow X(Z)$.

Proof. Since by Proposition 4.2 the domination mapping $Z^{*} \rightarrow \operatorname{Spec}(\operatorname{Kr}(Z))$ is a homeomorphism, it follows from the proposition that $X^{*}\left(Z^{*}\right) \rightarrow X(Z)$ is onto. To see that this mapping is one-to-one, suppose that $V, W \in Z$ such that $V$ and $W$ are centered on the same point of $X$, but $V^{*}$ and $W^{*}$ are centered on different points of $X^{*}$. Write $X=\bigcup_{i} \operatorname{Spec}\left(D_{i}\right)$. Then since $V$ and $W$ are centered on the same point of $X$, and $X^{*}=\bigcup_{i} \operatorname{Spec}\left(D^{*}\left[D_{i}\right]\right)$, it follows that there exists $i$ such that $V^{*}$ and $W^{*}$ are centered on different points of $\operatorname{Spec}\left(D^{*}\left[D_{i}\right]\right)$. Thus since $D^{*}\left[D_{i}\right] \subseteq D_{i}^{*}$, it must be that $V^{*}$ and $W^{*}$ are centered on different points of $D_{i}^{*}$, and there exist $f, g \in D_{i}[T]$ such that $c(g)=D_{i}$ and $f / g \in \mathfrak{M}_{V^{*}} \backslash \mathfrak{M}_{W^{*}}$. Then $g / f \in W^{*}$, so that since $c(g)=D_{i}$, we have $1 \in c(g) W^{*}=g W^{*} \subseteq f W^{*}=c(f) W^{*}$. Hence $c(f) \nsubseteq \subseteq \mathfrak{M}_{W^{*}}$. On the other hand, $f V^{*}=c(f) V^{*}$, so that $c(f) \subseteq \mathfrak{M}_{V}$. Therefore, $V$ and $W$ are centered on different points of $\operatorname{Spec}\left(D_{i}\right) \subseteq X$, a contradiction which implies the corollary.

In particular, the subspace $X^{*}\left(\mathfrak{X}^{*}\right)$ of $X^{*}$ is homeomorphic to $X$. 
Remark 4.5. Let $k$ be a field, let $F$ be an extension of $k$, and let $L$ be an extension of $F$. Denote by $\mathfrak{X}_{F / k}$ and $\mathfrak{X}_{L / k}$ the Zariski-Riemann spaces of $F / k$ and $L / k$, respectively. In [11, Theorem 1.4], Ducros shows that the map $\mathfrak{X}_{L / k} \rightarrow \mathfrak{X}_{F / k}: V \mapsto V \cap F$ is a continuous map that is, in our terminology, closed with respect to the inverse topology. Ducros also proves a graded version of this result [11, Theorem 1.5]. Another version of functoriality is given in [10, Proposition 4], where it is shown that when $R \subseteq S$ is an extension of domains, then the Kronecker fuction ring of $R$ embeds in that of $S$.

\section{Inverse closed subspaces of $\mathfrak{X}$}

In this section we consider in more detail inverse closed subpaces of $\mathfrak{X}$. We show first that these subspaces possess a locally ringed space structure inherited in an obvious way from $\mathfrak{X}$ and that with this structure, they are expressible as a projective limit of locally ringed inverse closed subspaces of projective models.

Let $X$ be a set, and let $\left\{A_{x}: x \in X\right\}$ be a collection of quasilocal rings with $D \subseteq A_{x} \subseteq F$. Then the Zariski topology on $X$ has as an open basis the sets of the form $\left\{x \in X: S \subseteq \bar{A}_{x}\right\}$, where $S$ is a finite subset of $F$ [45, Chapter VII, $\S 17]$. When $X$ is a subset of $\mathfrak{X}$ and $A_{V}=V$ for each $V \in X$, then this topology is precisely the subspace topology that $X$ inherits from $\mathfrak{X}$. Similarly, when $X$ is a subset of a projective model $Y$ of $F / D$ and $A_{x}=\mathcal{O}_{Y, x}$ for each $x \in X$, then this topology is the subspace topology on $X$.

In general, with $X$ a set and $\left\{A_{x}: x \in X\right\}$ a collection of quasilocal $D$-subalgebras over $F$, define a sheaf $\mathcal{O}_{X}$ on $X$ by $\mathcal{O}_{X}(\emptyset)=F$, and for each nonempty open subset $U$ of $X, \mathcal{O}_{X}(U)=\bigcap_{x \in U} A_{x}$, where for nonempty open sets $V \subseteq U$, the restriction map $\rho_{V}^{U}: \mathcal{O}_{X}(U) \rightarrow \mathcal{O}_{X}(V)$ is simply set inclusion. Moreover, for each $x \in X$, the stalk $\mathcal{O}_{X, x}$ of $\mathcal{O}_{X}$ at $x$ is $A_{x}$.

An inverse closed subset of a projective model is a spectral space (Proposition 3.1), as are the inverse closed subsets of $\mathfrak{X}$ (Proposition 2.7(1)). Thus we deduce the following lemma from the preceding discussion.

Lemma 5.1. If $X$ is a projective model of $F / D$, then every nonempty inverse closed subspace $Y$ of $X$ is a locally ringed spectral space with structure sheaf $\mathcal{O}_{Y}$ defined above. Similarily, every nonempty inverse closed subspace $Z$ of $\mathfrak{X}$ is a locally ringed spectral space with structure sheaf $\mathcal{O}_{Z}$.

In particular, when $Z$ is a subspace of $\mathfrak{X}$, then $\operatorname{inv}(X(Z))$ is a locally ringed space. Implicit in the next theorem (and explicit in its proof) is the fact that when $\mathfrak{S}$ is a dominant system of projective models of $F / D$, then $\{\operatorname{inv}(X(Z)): X \in \mathfrak{S}\}$ is a projective system of locally ringed spaces.

Theorem 5.2. Let $Z$ be a subspace of $\mathfrak{X}$, and let $\mathfrak{S}$ be a dominant system of projective models of $F / D$. Then, as locally ringed spaces,

$$
\operatorname{inv}(Z)=\lim _{\longleftarrow} \operatorname{inv}(X(Z)),
$$

where $X$ ranges over $\mathfrak{S}$. 
Proof. For each $X \in \mathfrak{S}$, let $X^{\prime}=\operatorname{inv}(X(Z))$. By Lemma 5.1, $X^{\prime}$ is a locally ringed space with structure sheaf $\mathcal{O}_{X^{\prime}}$. Let $X$ and $Y$ be projective models in $\mathfrak{S}$ such that $Y$ dominates $X$, and let $\delta=\left(d, d^{\#}\right): Y \rightarrow X$ be the domination morphism. Define a morphism $\phi=\left(f, f^{\#}\right): Y^{\prime} \rightarrow X^{\prime}$ in the following way. Let $f$ be the restriction of $d$ to $Y^{\prime}$, so that $f$ is a continuous map from $Y^{\prime}$ to $X$. We claim that $f\left(Y^{\prime}\right) \subseteq X^{\prime}$. Now $d$ is a spectral map (for as a proper morphism, $\delta$ is quasicompact), and hence by Lemma 2.7 (2), $f\left(Y^{\prime}\right)=d(\operatorname{inv}(Y(Z))) \subseteq$ $\operatorname{inv}(d(Y(Z)))=\operatorname{inv}(X(Z))=X^{\prime}$, which shows that $f$ is a continuous map from $Y^{\prime}$ into $X^{\prime}$. Next, define the sheaf morphism $f^{\#}: \mathcal{O}_{X^{\prime}} \rightarrow f_{*} \mathcal{O}_{Y^{\prime}}$ for each open set $U$ of $X^{\prime}$ by $f_{U}^{\#}: \mathcal{O}_{X^{\prime}}(U) \rightarrow \mathcal{O}_{Y^{\prime}}\left(d^{-1}(U)\right): s \mapsto s$. This makes sense because $\mathcal{O}_{X^{\prime}}(U) \subseteq \mathcal{O}_{Y^{\prime}}\left(d^{-1}(U)\right)$. If $y \in Y^{\prime}$, then the stalk of $Y^{\prime}$ at $y$ is $\mathcal{O}_{Y^{\prime}, y}=\mathcal{O}_{Y, y}$, and from this it follows that since $\delta$ is a morphism of locally ringed spaces, then so is $\phi$. This shows that $\left\{X^{\prime}: X \in \mathfrak{S}\right\}$ is a projective system of locally ringed spaces. Now by Lemma 2.8 $\operatorname{inv}(Z)$ is the projective limit of the topological spaces $X^{\prime}=\operatorname{inv}(X(Z)$ ), where $X$ ranges over $\mathfrak{S}$. Moreover, it follows from Proposition 3.3 (3) and the fact that the structure sheaf on each locally ringed space $X^{\prime}$ is induced by the structure sheaf on $X$ that $\operatorname{inv}(Z)$ is the projective limit of $\left\{X^{\prime}: X \in \mathfrak{S}\right\}$ in the category of locally ringed spaces.

Remark 5.3. Though it is a locally ringed space, an inverse closed subspace of a projective model of $F / D$ need not be a scheme. For example, when $D=k\left[T_{0}, T_{1}\right]$, with $k$ a field and $T_{0}$ and $T_{1}$ indeterminates for $k$, then the set $X$ of all prime ideals of $D$ of height $\leq 1$ is inverse closed in the projective model $\operatorname{Spec}(D)$ (since it is closed under generalizations and all open subsets of $\operatorname{Spec}(D)$ are quasicompact), but $X$ is not a scheme with respect to the structure sheaf $\mathcal{O}_{X}$. For suppose $U$ is a nonempty open subset of $\operatorname{Spec}(D)$ such that $X \cap U$ is an affine scheme. Then $X \cap U$ contains all but at most finitely many height one prime ideals of $D$, and hence since $D$ is a UFD there is $f \in D$ such that $X \cap U=\{P \in \operatorname{Spec}(D): f \notin P\}$. But then the ring of global sections of $X \cap U$ is $D_{f}$, and this ring has Krull dimension 2, while the stalks of $X \cap U$ all have dimension one. So $X$ cannot be a scheme.

The quasicompact open sets in $\mathfrak{X}$ are of the form $\mathfrak{X}_{D_{1}} \cup \cdots \cup \mathfrak{X}_{D_{n}}$, where $D_{1}, \ldots, D_{n}$ are finitely generated $D$-subalgebras of $F$. One of the advantages of working with affine subsets of $\mathfrak{X}$ is that it follows from Proposition 5.6 that when $Z$ is affine, then $Z$ is inverse closed if and only if $Z=\mathfrak{X}_{R}$ for some ring $R$ with quotient field $F$. That a subset of the form $\mathfrak{X}_{R}$ is inverse closed is always true, but the converse, that an inverse closed set has this form, requires additional hypotheses, such as that $Z$ is affine. For example, consider the case where $D$ is a local Noetherian UFD with quotient field $F$ and Krull dimension $>1$. Let $p$ be a prime element of $D$. Then $\mathfrak{X}_{D[1 / p]} \cup \mathfrak{X}_{D_{(p)}}$ is an inverse closed subset of $\mathfrak{X}$ that is not of the form $\mathfrak{X}_{R}$ for any overring $R$ of $D$. As statement (3) of the next lemma shows, such examples do not arise for affine subspaces.

Lemma 5.4. Suppose $D$ is a Prüfer domain with quotient field $F$.

(1) If $S$ and $T$ are $D$-submodules of $F$, then $\mathfrak{X}_{S \cap T}=\mathfrak{X}_{S} \cup \mathfrak{X}_{T}$.

(2) A subset of $\mathfrak{X}$ is open and quasicompact in the Zariski topology if and only if it is of the form $\mathfrak{X}_{S}$ for some finite subset $S$ of $F$.

(3) A subset of $\mathfrak{X}$ is inverse closed in $\mathfrak{X}$ if and only if it is of the form $\mathfrak{X}_{S}$ for some overring $S$. 
Proof. (1) Let $A=S \cap T$, and let $V \in \mathfrak{X}$. Then since $D$ is a Prüfer domain, $V=D_{P}$ for some prime ideal $P$ of $D$. Hence $A_{P}=S_{P} \cap T_{P}$, and since $D_{P}$ is a valuation domain, this forces $A_{P}=S_{P}$ or $A_{P}=T_{P}$. Thus $S \subseteq V$ or $T \subseteq V$.

(2) Let $Z$ be a quasicompact open subset of $\mathfrak{X}$. Then since the subsets of $\mathfrak{X}$ of the form $\mathfrak{X}_{S}$, with $S$ a finite subset of $F$, constitute a basis of open subsets of $\mathfrak{X}$ in the Zariski topology, there exist finite subsets $S_{1}, \ldots, S_{n}$ of $F$ such that $Z=\mathfrak{X}_{S_{1}} \cup \cdots \cup \mathfrak{X}_{S_{n}}$. For each $i=1, \ldots, n$, let $I_{i}$ be the fractional ideal of $D$ generated by $S_{i}$. Then by $(1), Z=\mathfrak{X}_{I_{1} \cap \cdots \cap I_{n}}$. Statement (2) now follows from the fact that since $D$ is a Prüfer domain, the intersection of the finitely generated fractional ideals $I_{1}, \ldots, I_{n}$ is a finitely generated fractional ideal [18, Proposition 21.4].

(3) Suppose $Z$ is inverse closed in $\mathfrak{X}$. Then by (2), $Z=\bigcap_{i} \mathfrak{X}_{D_{i}}$ for a collection $\left\{D_{i}\right\}$ of overrings of $R$, and hence $Z=\mathfrak{X}_{S}$, where $S$ is the overring of $R$ generated by the rings $D_{i}$. Conversely, if $S$ is an overring of $D$, then $\mathfrak{X}_{S}=\bigcap_{s \in S} \mathfrak{X}_{\{s\}}$, so that by (2), $\mathfrak{X}_{S}$, as an intersection of inverse closed subsets, is inverse closed.

Remark 5.5. A domain $D$ with quotient field $F$ is vacant if it has a unique Kronecker function ring (for an explanation of the notion of a Kronecker function ring associated to a domain, see the discussion before Proposition 5.10). Fabbri has shown that these domains are characterized by a version of property (1) in the proposition: $D$ is vacant if and only if $\mathfrak{X}=\mathfrak{X}_{D_{1}} \cup \cdots \cup \mathfrak{X}_{D_{n}}$ whenever $D=D_{1} \cap D_{2} \cap \cdots \cap D_{n}$ [12, Theorem 3.1]. It is an open question as to whether statement (1) is in general equivalent to the property of being vacant [12, p. 1075].

The description of the inverse closure of non-affine subsets in $\mathfrak{X}$ is less transparent, but the Kronecker function ring construction discussed in the last section is useful here too in clarifying things. Here, as in Section $3, \mathfrak{X}_{R}^{*}$ is the set of valuation rings in $\mathfrak{X}^{*}$ containing $R$.

Proposition 5.6. Let $Z$ be a subspace of $\mathfrak{X}$, and let $A=\bigcap_{V \in Z} V$. Then the following statements hold for $Z$ and $A$.

(1) $Z=\operatorname{inv}(Z)$ if and only if $Z^{*}=\mathfrak{X}_{\operatorname{Kr}(Z)}^{*}$.

(2) $\operatorname{inv}(Z)=\left\{V \cap F: V \in \mathfrak{X}_{\operatorname{Kr}(Z)}^{*}\right\}$

(3) $(\operatorname{inv}(Z))^{*}=\mathfrak{X}_{\mathrm{Kr}(Z)}^{*}$.

(4) $A=\bigcap_{V \in \operatorname{inv}(Z)} V$.

(5) When $Z$ is affine, then $\operatorname{inv}(Z)=\mathfrak{X}_{A}$.

(6) $(\operatorname{inv}(Z))^{*}=\operatorname{inv}\left(Z^{*}\right)$.

Proof. (1) Suppose $Z$ is inverse closed in $\mathfrak{X}$. Then by Proposition 4.2, $Z^{*}$ is inverse closed in $\mathfrak{X}^{*}$. Moreover, $Z^{*} \subseteq \mathfrak{X}_{\operatorname{Kr}(Z)}^{*}$, so since $\operatorname{Kr}(Z)$ is a Prüfer domain with quotient field $F^{*}$, we have by Lemma [5.4(3) that $Z^{*}=\mathfrak{X}_{R}^{*}$ for some overring $R$ of $\operatorname{Kr}(Z)$. As an overring of a Prüfer domain, $R$ is itself a Prüfer domain, and in particular, it is the intersection of its valuation rings, so we conclude that $R=\operatorname{Kr}(Z)$. Therefore, $Z^{*}=\mathfrak{X}_{\operatorname{Kr}(Z)}^{*}$. Conversely, if $Z^{*}=\mathfrak{X}_{\operatorname{Kr}(Z)}^{*}$, then $Z^{*}$ is by Lemma $5.4(3)$ inverse closed in $\mathfrak{X}^{*}$. Thus by Proposition $4.2, Z$ is inverse closed in $\mathfrak{X}$. 
(2) By Lemma $5.4(3), \mathfrak{X}_{\operatorname{Kr}(Z)}^{*}$ is an inverse closed subset of $\mathfrak{X}^{*}$, so by Proposition 4.2 $\left\{V \cap F: V \in \mathfrak{X}_{\operatorname{Kr}(Z)}^{*}\right\}$ is an inverse closed subset of $\mathfrak{X}$. Thus to prove that $\operatorname{inv}(Z)=\{V \cap F$ : $\left.V \in \mathfrak{X}_{\operatorname{Kr}(Z)}^{*}\right\}$, it suffices to show that every inverse closed subset of $\mathfrak{X}$ containing $Z$ contains also $\left\{V \cap F: V \in \mathfrak{X}_{\operatorname{Kr}(Z)}^{*}\right\}$. If $Z^{\prime}$ is another inverse closed subset of $\mathfrak{X}$ containing $Z$, then by $(1),\left(Z^{\prime}\right)^{*}=\mathfrak{X}_{\operatorname{Kr}\left(Z^{\prime}\right)}^{*}$. Thus $\left\{V \cap F: V \in \mathfrak{X}_{\operatorname{Kr}(Z)}^{*}\right\} \subseteq\left\{V \cap F: V \in\left(Z^{\prime}\right)^{*}\right\}=Z^{\prime}$. Also, since $Z \subseteq Z^{\prime}$, we have $\mathfrak{X}_{\operatorname{Kr}(Z)}^{*} \subseteq \mathfrak{X}_{\operatorname{Kr}\left(Z^{\prime}\right)}^{*}$, so that $\left\{V \cap F: V \in \mathfrak{X}_{\mathrm{Kr}(Z)}^{*}\right\} \subseteq Z^{\prime}$. This verifies (2).

(3) This follows from (2) and Proposition 4.2 .

(4) It is enough by (2) to observe that $\mathfrak{X}_{\operatorname{Kr}(Z)}^{*}=\mathfrak{X}_{\operatorname{Kr}(\operatorname{inv}(Z))}^{*}$, which is the case by (3).

(5) Since $Z$ is affine, $A$ is a Prüfer domain with quotient field $F$. Thus by Lemma 5.4(3), $\operatorname{inv}(Z)=\mathfrak{X}_{R}$ for some ring $R$ between $D$ and $F$ that is integrally closed in $F$. By (4), $A=\bigcap_{V \in \operatorname{inv}(Z)} V=\bigcap_{V \in \mathfrak{X}_{R}} V=R$, so $\operatorname{inv}(Z)=\mathfrak{X}_{A}$.

(6) By (3) and (5), $\operatorname{inv}\left(Z^{*}\right)=\mathfrak{X}_{\operatorname{Kr}(Z)}^{*}=(\operatorname{inv}(Z))^{*}$.

A consequence of the proposition is that localizations of $A=\bigcap_{V \in Z} V$ can be represented with subsets of $\operatorname{inv}(Z)$.

Corollary 5.7. If $Z \subseteq \mathfrak{X}$ and $S$ is a multiplicatively closed subset of $A=\bigcap_{V \in Z} V$, then there exists $Y \subseteq \operatorname{inv}(Z)$ such that $A_{S}=\bigcap_{V \in Y} V$.

Proof. Since $A=\operatorname{Kr}(Z) \cap F$, we have $A_{S}=\operatorname{Kr}(Z)_{S} \cap F$. Moreover, $\mathfrak{X}_{\operatorname{Kr}(Z)_{S}}^{*} \subseteq \mathfrak{X}_{\operatorname{Kr}(Z)}^{*}$, so by Proposition 5.6(3), there is a subset $Y$ of $\operatorname{inv}(Z)$ such that $Y^{*}=\mathfrak{X}_{\operatorname{Kr}(Z)_{S}}^{*}$. Thus $\bigcap_{V \in Y} V=\operatorname{Kr}(Y) \cap F=\operatorname{Kr}(Z)_{S} \cap F=A_{S}$.

The proposition also yields an interpretation of $\mathrm{pt}(Z)$.

Corollary 5.8. Let $Z$ be a subspace of $\mathfrak{X}$. The continuous map,

$$
g: \operatorname{Spec}(\operatorname{Kr}(Z)) \rightarrow \mathfrak{X}: P \mapsto \operatorname{Kr}(Z)_{P} \cap F
$$

restricts to a homeomorphism of $\operatorname{Max}(\operatorname{Kr}(Z))$ onto $\mathrm{pt}(Z)$.

Proof. Let $M \in \operatorname{Max}(\operatorname{Kr}(Z))$. Then by Proposition $5.6(2), g(M)=\operatorname{Kr}(Z)_{M} \cap F \in \operatorname{inv}(Z)$. Moreover, the mapping $g$ is by Proposition 4.2 a closed map, so since $M$ is a closed point in $\operatorname{Max}(\operatorname{Kr}(Z)), g(M) \in \operatorname{pt}(Z)$. Thus $g$ carries $\operatorname{Max}(\operatorname{Kr}(Z)))$ into $\operatorname{pt}(Z)$. Now let $V \in$ $\operatorname{pt}(Z)$. Then Proposition [5.6(3) implies that $V^{*} \in \mathfrak{X}_{\mathrm{Kr}(Z)}^{*}$, and hence there is a prime ideal $P$ of $\operatorname{Kr}(Z)$ such that $V^{*}=\operatorname{Kr}(Z)_{P}$. By Proposition 4.2, the mapping $g$ induces a homeomorphism of $\operatorname{Spec}(\operatorname{Kr}(Z))$ onto $\left\{V \cap F: V \in \mathfrak{X}_{\mathrm{Kr}(Z)}^{*}\right\}$. Since $V$ is a closed point in $\operatorname{inv}(Z)=\left\{V \cap F: V \in \mathfrak{X}_{\operatorname{Kr}(Z)}^{*}\right\}$, then $V^{*}$ is a closed point in $\mathfrak{X}_{\operatorname{Kr}(Z)}^{*}$, so that $P$ is a closed point in $\operatorname{Spec}(\operatorname{Kr}(Z))$. As such, $P \in \operatorname{Max}(\operatorname{Kr}(Z))$, so that $g$ carries $\operatorname{Max}(\operatorname{Kr}(Z))$ onto pt $(Z)$. By Proposition 4.2, $g$ is a closed continuous map, and the proposition follows

We mention several examples of inverse closed subsets. Some explicit interpretations of $\operatorname{inv}(Z)$ are also given in [33] in the case where $D$ is a two-dimensional Noetherian domain with quotient field $F$.

\section{Example 5.9.}


(1) Let $f(T)$ be a nonconstant monic polynomial in $D[T]$, and let $Z$ be the set of valuation rings $V$ in $\mathfrak{X}$ such that $f$ has no root in the residue field of $V$. If $Z$ is nonempty, then as noted in Example 4.1(2), $Z$ is affine. Moreover, with $A=\bigcap_{V \in Z} V$, then $Z=\mathfrak{X}_{A}$. For suppose $V \in \mathfrak{X}_{A}$ and $f(x) \in \mathfrak{M}_{V}$ for some $x \in V$. If $f(x)=0$, then $x$ is in the integral closure of $D$ in $F$, and hence an element of each $V$ in $Z$, a contradiction to the fact that $f$ has no root in the residue field of $V$. Thus $f(x) \neq 0$ and since $f(x) \in \mathfrak{M}_{V}$, then $f(x)^{-1} \notin V$, so that $f(x)^{-1} \notin A$. But then $f(x)^{-1} \notin W$ for some $W \in Z$, so that since $W$ is a valuation ring, $f(x) \in \mathfrak{M}_{W}$, a contradiction. Thus by Proposition [5.6(5), $Z=\operatorname{inv}(Z)$.

(2) Suppose that $D$ is a Krull domain with quotient field $F$, and let $Z$ be the set of essential prime divisors of $D$, i.e., $Z$ is the set of all localizations of $D$ at height 1 prime ideals of $D$. Then $\operatorname{Kr}(Z)$ is a Dedekind domain with quotient field $F^{*}$, and hence $\mathfrak{X}_{\operatorname{Kr}(Z)}^{*}=Z^{*} \cup\left\{F^{*}\right\}$. Thus by Proposition 5.6 (3), $\operatorname{inv}(Z)=Z \cup\{F\}$. Moreover, if $D$ has Krull dimension $>1$, then $Z$ is not affine. This example is a special case of the more general fact that the inverse closure of a Noetherian subspace $Z$ of $\mathfrak{X}$ is the closure of $Z$ under generalizations (Corollary 2.3).

It follows from Proposition 5.6(5) that two affine subspaces $Z_{1}$ and $Z_{2}$ in $\mathfrak{X}$ have the same inverse closure if and only if $\bigcap_{V \in Z_{1}} V=\bigcap_{V \in Z_{2}} V$. For non-affine subspaces we can assert via Proposition 5.6(4) only that when $Z_{1}$ and $Z_{2}$ have the same inverse closure, then $\bigcap_{V \in Z_{1}} V=\bigcap_{V \in Z_{2}} V$. The reason that the converse of this is generally not true is that an integrally closed domain can have more than one Kronecker function ring associated to it. To clarify this, we say that for a ring $R$ between $D$ and $F$ that is integrally closed in $F$, an overring $S$ of $\operatorname{Kr}(\mathfrak{X})$ is a Kronecker F-function ring of $R$ if $R=S \cap F$. (This is a special case of the class of $F$-function rings introduced by Halter-Koch in [20]). Thus $\operatorname{Kr}\left(\mathfrak{X}_{R}\right)$ is the smallest Kronecker $F$-function ring of $R$, and when $R$ is a Prüfer domain with quotient field $F$, it is the unique Kronecker $F$-function ring of $R$ [18, Theorem 26.18]. In general the property of having a unique Kronecker $F$-function ring does not characterize Prüfer domains [12]. In any case, the next proposition shows that the complexity (or lack thereof) of the class of Kronecker $F$-function rings of a given domain $R$ explains the complexity of the class of inverse closed subspaces of $\mathfrak{X}$ that represent $R$.

Proposition 5.10. Let $R$ be a ring between $D$ and $F$ that is integrally closed in $F$. Then there is a one-to-one correspondence between the Kronecker F-function rings $S$ of $R$ and inverse closed subspaces $Z$ of $\mathfrak{X}$ such that $R=\bigcap_{V \in Z} V$. The correspondence is given by

$$
S \mapsto\left\{V \cap F: V \in \mathfrak{X}_{S}^{*}\right\} \quad \text { and } \quad Z \mapsto \operatorname{Kr}(Z) .
$$

Proof. Let $Z$ be a closed subspace of $\mathfrak{X}$ such that $R=\bigcap_{V \in Z} V$. Then by Proposition $5.6(2)$, $Z=\left\{V \cap F: V \in \mathfrak{X}_{\operatorname{Kr}(Z)}^{*}\right\}$, so $\operatorname{Kr}(Z)$ is a Kronecker $F$-function ring of $R$. Also, if $S$ and $T$ are Kronecker $F$-function rings of $R$ with $\left\{V \cap F: V \in \mathfrak{X}_{S}^{*}\right\}=\left\{V \cap F: V \in \mathfrak{X}_{T}^{*}\right\}$, then $S=\bigcap_{V \in \mathfrak{X}_{S}} V=\operatorname{Kr}\left(\left\{V \cap F: V \in \mathfrak{X}_{S}^{*}\right\}\right)=\operatorname{Kr}\left(\left\{V \cap F: V \in \mathfrak{X}_{T}^{*}\right\}\right)=\bigcap_{V \in \mathfrak{X}_{T}} V=T$. This proves the proposition.

By way of example, every Krull domain of dimension at least 2 has more than one Kronecker function ring; see [12, Proposition 2.10]. 


\section{Affine schemes in $\mathfrak{X}$}

In this section we characterize the subspaces of $\mathfrak{X}$ that are affine schemes. As discussed at the beginning of Section 5 , whether a subspace of $\mathfrak{X}$ when equipped with the natural choice of a structure presheaf is a locally ringed subspace can be detected topologically, in the sense that this presheaf is a sheaf if and only if the subspace is irreducible. In contrast, whether a subspace is an affine scheme cannot be detected topologically since $\mathfrak{X}$ is homeomorphic to the affine scheme $\mathfrak{X}^{*}$, regardless of the choice of $D$ and $F$. Our first characterization of affine schemes in $\mathfrak{X}$ is the following theorem. In the theorem, $\mathcal{O}_{Z}$ is the presheaf defined at the beginning of Section 5 as $\mathcal{O}_{Z}(U)=\bigcap_{V \in U} V$ for all nonempty open subsets $U$ of $Z$.

Theorem 6.1. Let $Z$ be a subspace of $\mathfrak{X}$. Then $\left(Z, \mathcal{O}_{Z}\right)$ is an affine scheme if and only if $Z$ is inverse closed in $\mathfrak{X}$ and $\mathcal{O}_{Z}(Z)$ is a Prüfer domain with quotient field $F$.

Proof. Let $A=\mathcal{O}_{Z}(Z)=\bigcap_{V \in Z} V$, and suppose that $\left(Z, \mathcal{O}_{Z}\right)$ is an affine scheme. Then by assumption, $A_{P} \in Z$ for each prime ideal $P$ of $A$. In particular, each localization of $A$ at a prime ideal is a valuation domain with quotient field $F$, and hence $A$ is a Prüfer domain with quotient field $F$. Moreover, if $V \in Z$, then since $Z$ is an affine scheme, $V=A_{P}$ for some prime ideal $P$ of $A$, so that $Z=\mathfrak{X}_{A}$. By Proposition 5.6(1), $\mathfrak{X}_{A}$ is inverse closed.

Conversely, suppose that $A$ is a Prüfer domain with quotient field $F$ and $Z$ is inverse closed. By Proposition 2.5, $Z$ is irreducible, and hence $\mathcal{O}_{Z}$ is a sheaf. Since $A$ is a Prüfer domain, then each $V \in Z$ is a localization of $A$ at a prime ideal. Also, by Proposition 5.6(4), $Z=\operatorname{inv}(Z)=\mathfrak{X}_{A}$, so it follows that each localization of $A$ at a prime ideal is in $Z$. Thus $Z$ can be identified with $\operatorname{Spec}(A)$, and $\left(Z, \mathcal{O}_{Z}\right)$ is an affine scheme.

Corollary 6.2. The Zariski-Riemann space $\mathfrak{X}$ is an affine scheme if and only if the integral closure of $D$ in $F$ is a Prüfer domain with quotient field $F$.

More generally, the inverse closed subsets of $\mathfrak{X}$ correspond to affine schemes in $\mathfrak{X}^{*}$ :

Corollary 6.3. A nonempty subset $Z$ of $\mathfrak{X}$ is inverse closed if and only if $\left(Z^{*}, \mathcal{O}_{Z^{*}}\right)$ is an affine scheme.

Proof. If $Z$ is inverse closed, then by Proposition 4.2. $Z^{*}$ is also inverse closed, and hence by Proposition 5.6 (3), $Z^{*}=\mathfrak{X}_{\mathrm{Kr}(Z)}^{*}$, so that by Corollary 6.2, $\left(Z^{*}, \mathcal{O}_{Z^{*}}\right)$ is an affine scheme. Conversely, if $\left(Z^{*}, \mathcal{O}_{Z^{*}}\right)$ is an affine scheme, then by Theorem 6.1, $Z^{*}$ is inverse closed, and hence by Proposition 4.2, $Z$ is inverse closed.

Motivated by the terminology of [45, Chapter VII, §17], we define an affine model $X$ of $F / D$ to be an affine integral scheme of finite type over $D$ whose function field is a subfield of $F$; equivalently, there is a finitely generated $D$-subalgebra $R$ of $F$ such that $X=\left\{R_{P}: P \in \operatorname{Spec}(R)\right\}$.

Corollary 6.4. A nonempty subset $Z$ of $\mathfrak{X}$ is affine if and only if $\operatorname{inv}(Z)$ is the projective limit of a projective system of affine models of $F / D$.

Proof. Suppose that $Z$ is affine. Then by definition, $A=\bigcap_{V \in Z} V$ is a Prüfer domain with quotient field $F$. Since by Proposition 5.6(4), $A=\bigcap_{V \in \operatorname{inv}(\mathrm{Z})} V$, then by Theorem6.1, inv $(Z)$ is an affine scheme, so that by Proposition [5.6(5), the morphism $\operatorname{inv}(Z) \rightarrow \operatorname{Spec}(A)$ that 
sends a valuation $\operatorname{ring} \operatorname{in} \operatorname{inv}(Z)$ to its center on $A$ is an isomorphism of schemes. Since also $A$ is the direct limit of finitely generated $D$-subalgebras, it follows that $\operatorname{inv}(Z)$ is the projective limit of the projective system of affine models dominated by $\operatorname{inv}(Z)$. Conversely, a projective limit of affine schemes is necessarily affine; see for example [41, Lemma 01YW].

We give next a more refined version of Corollary 6.4 by showing that whether a subset $Z$ of $\mathfrak{X}$ is affine is determined by whether every projective model is dominated by an affine model that is in turn dominated by $Z$. This is a consequence of the following routine lemma, whose proof we omit.

Lemma 6.5. Let $A$ be a domain with quotient field $F$, and let $t_{0}, \ldots, t_{n}$ be nonzero elements in $F$. Then $\left(t_{0}, \ldots, t_{n}\right) A$ is an invertible fractional ideal of $A$ if and only if

$$
A=\sum_{0=1}^{n} t_{i}:_{A}\left(t_{0}, \ldots, t_{n}\right) A .
$$

Theorem 6.6. The following are equivalent for a subset $Z$ of $\mathfrak{X}$.

(1) $Z$ is affine.

(2) For every projective model $X$ of $F / D$, there exists an affine model $Y$ of $F / D$ dominating $X$ and dominated by $Z$.

(3) For every projective model $X$ of $F / D$ defined by 2 elements of $F$, there exists an affine model $Y$ of $F / D$ dominating $X$ and dominated by $Z$.

Proof. $(1) \Rightarrow(2)$ Suppose that $Z$ is an affine subset of $\mathfrak{X}$, and let $X$ be a projective model of $F / D$ defined by $t_{0}, \ldots, t_{n} \in F$ (as in Section 3). Let $A=\bigcap_{V \in Z} V$. Then since $A$ is a Prüfer domain, $\left(t_{0}, \ldots, t_{n}\right) A$ is an invertible fractional ideal of $A$, so we have by Lemma 6.5 that

$$
A=\sum_{0=1}^{n} t_{i}:_{A}\left(t_{0}, \ldots, t_{n}\right) A .
$$

Thus there exist $b_{1}, \ldots, b_{n} \in A$ and $a_{i j} \in A, i, j=1, \ldots, n$, such that

(†) $\quad 1=b_{1}+\cdots+b_{n}$ and for each $i, b_{i}=a_{i 1} \frac{t_{i}}{t_{1}}=\cdots=a_{i n} \frac{t_{i}}{t_{n}}$.

Define $R=D\left[\left\{b_{1}, \ldots, b_{n}\right\} \cup\left\{a_{i j}: i, j=1, \ldots, n\right\}\right]$. Then $R$ is a finitely generated $D$ subalgebra of $A$. Let $Y=\left\{R_{P}: P \in \operatorname{Spec}(R)\right\}$, so that $Y$ is an affine submodel of $F / D$. Since $R \subseteq A$, then $Z$ dominates $Y$. We claim that $Y$ dominates $X$. Indeed, let $P$ be a prime ideal of $R$. Observe that from $(\dagger)$ and the definition of $R$, it follows that $R=\sum_{0=1}^{n} t_{i}:_{R}$ $\left(t_{0}, \ldots, t_{n}\right) R$. Thus there exists $i=1, \ldots, n$ such that $R_{P}=t_{i}:_{R_{P}}\left(t_{0}, \ldots, t_{n}\right) R_{P}$. Consequently, $D\left[\frac{t_{0}}{t_{i}}, \ldots, \frac{t_{n}}{t_{i}}\right] \subseteq R_{P}$, and since the projective model $X$ is defined by $t_{0}, \ldots, t_{n}$, we conclude that $R_{P}$ dominates a local ring in $X$. Therefore, $Y$ dominates $X$.

$(2) \Rightarrow(3)$ This is clear.

$(3) \Rightarrow(1)$ Assuming (3), we claim that $A=\bigcap_{V \in Z} V$ is a Prüfer domain with quotient field $F$. It suffices to show that for each prime ideal $P$ of $A$ and $0 \neq t \in F, t$ or $t^{-1} \in A_{P}$. Let $P$ be a prime ideal of $A$ and let $0 \neq t \in F$. Let $X$ be the projective model of $F / D$ defined by $\{1, t\}$. By assumption there is a finitely generated $D$-subalgebra $R$ of $F$ such 
that $Z$ dominates the affine model $Y=\left\{R_{Q}: Q \in \operatorname{Spec}(R)\right\}$ (equivalently, $R \subseteq A$ ) while $Y$ dominates $X$. Since $Y$ dominates $X$ and $X$ consists of the localizations of $D[t]$ and $D\left[t^{-1}\right]$ at prime ideals, it follows that $R_{P \cap R}$, hence $A_{P}$, contains either $t$ or $t^{-1}$. Since the choice of $t$ was arbitrary, we conclude that $A_{P}$ is a valuation domain with quotient field $F$, and hence $A$ is a Prüfer domain with quotient field $F$.

Corollary 6.7. A domain $A$ with quotient field $F$ is a Prüfer domain if and only if every projective model of $F / A$ is affine.

Proof. If $A$ is a Prüfer domain and $X$ is a projective model of $F / A$, then $X$ consists of valuation rings between $A$ and $F$, and hence each member of $X$ is a localization of $A$. Also, if $V$ is a valuation ring between $A$ and $F$, then $V$ dominates $\mathcal{O}_{X, x}$ for some $x \in X$. But $\mathcal{O}_{X, x}$ is a valuation domain, so it follows that $V=\mathcal{O}_{X, x} \in X$. Thus $X=\operatorname{Spec}(A)$. Conversely, if every projective model of $F / A$ is affine, then by Theorem 6.6, $A$ is a Prüfer domain.

Acknowledgement. I thank Marco Fontana for pointing out to me a mistake in an earlier version of the article.

\section{References}

[1] E. Becker, The real holomorphy ring and sums of $2 n$th powers. In Real algebraic geometry and quadratic forms (Rennes, 1981), Lecture Notes in Math. 959, 139-181. Springer, Berlin, 1982.

[2] R. Berr, On real holomorphy rings. In Real analytic and algebraic geometry (Trento, 1992), 47-66. de Gruyter, Berlin, 1995.

[3] N. Bourbaki, Commutative algebra, Chapters 1-7, Springer, 1985.

[4] N. Bourbak, General topology, Chapters 1-4, Springer, 1998.

[5] L. Brocker and H. Schülting, Valuations of function fields from the geometrical point of view, J. Reine Angew. Math. 365 (1986), 12-32.

[6] M. A. Buchner and W. Kucharz, On relative real holomorphy rings, Manuscripta Math., 63 (1989), 303-316.

[7] P. J. Cahen and J. L. Chabert, Integer-valued polynomials, Mathematical Surveys and Monographs, 48. American Mathematical Society, Providence, RI, 1997.

[8] B. Conrad and M. Temkin, Non-Archimedean analytification of algebraic spaces, J. Alg. Geom. 18 (2009), 731-788.

[9] D. Dobbs, R. Fedder and M. Fontana, Abstract Riemann surfaces of integral domains and spectral spaces. Ann. Mat. Pura Appl. (4) 148 (1987), 101-115.

[10] D. Dobbs and M. Fontana, Kronecker function rings and abstract Riemann surfaces. J. Algebra 99 (1986), no. 1, 263-274. 
[11] A. Ducros, Flatness in non-Archimedean analytic geometry, arXiv:1107.4259v1.

[12] A. Fabbri, Integral domains having a unique Kronecker function ring, J. Pure Appl. Algebra 215 (2011), no. 5, 1069-1084.

[13] A. Fabbri and O. Heubo-Kwegna, Projective star operations and graded domains, J. Commutative Alg., to appear.

[14] C. Favre, Countability properties of Berkovich spaces, arXiv:1103.6233v1.

[15] C. Favre and M. Jonsson, The valuative tree. Lecture Notes in Mathematics, 1853. Springer-Verlag, Berlin, 2004.

[16] C. Finocchiaro, M. Fontana and K. A. Loper, The constructible topology on the spaces of valuation domains, preprint.

[17] M. Fontana, J. Huckaba, and I. Papick, Prüfer Domains, Marcel Dekker, New York, 1997.

[18] R. Gilmer, Multiplicative ideal theory, Queen's Papers in Pure and Applied Mathematics, No. 12, Queen's University, Kingston, Ont. 1968.

[19] R. Gilmer and W. Heinzer, Primary ideals with finitely generated radical in a commutative ring, Manuscripta Math. 78 (1993), 201-221.

[20] F. Halter-Koch, Kronecker function rings and generalized integral closures, Comm. Algebra 31 (2003), no. 1, 45-59.

[21] R. Hartshorne, Algebraic geometry. Graduate Texts in Mathematics, No. 52. SpringerVerlag, New York-Heidelberg, 1977.

[22] W. Heinzer, C. Rotthaus and J. Sally, Formal fibers and birational extensions, Nagoya Math. J. 131 (1993), 1-38.

[23] O. Heubo-Kwegna, Kronecker function rings of transcendental field extensions, Comm. Algebra 38 (2010), no. 8, 2701-2719.

[24] M. Hochster, Prime ideal structure in commutative rings, Trans. Amer. Math. Soc. 142 (1969), 43-60.

[25] R. Huber and M. Knebusch, On valuation spectra. Recent advances in real algebraic geometry and quadratic forms (Berkeley, CA, 1990/1991; San Francisco, CA, 1991), 167-206, Contemp. Math., 155, Amer. Math. Soc., Providence, RI, 1994.

[26] P. Johnstone, Stone spaces, Reprint of the 1982 edition. Cambridge Studies in Advanced Mathematics, 3. Cambridge University Press, Cambridge, 1986.

[27] B. Kahn and R. Sujatha, Birational geometry and localisation of categories, arXiv:0805.3753v1.

[28] F.-V. Kuhlmann, Places of algebraic function fields in arbitrary characteristic, Adv. Math., 188 (2004), no. 2, 399-424. 
[29] F.-V. Kuhlmann, Value groups, residue fields, and bad places of rational function fields, Trans. Amer. Math. Soc. 356 (2004), no. 11, 4559-4600.

[30] M. Nagata, Local rings, Interscience Tracts in Pure and Applied Mathematics, No. 13, John Wiley \& Sons, New York-London 1962.

[31] J. Ohm and R. L. Pendleton, Rings with noetherian spectrum, Duke Math. J. 35 (1968), 631-639.

[32] B. Olberding, Holomorphy rings in function fields, in Multiplicative ideal theory in commutative algebra, 331-348, Springer-Verlag, 2006.

[33] B. Olberding, Quasi-Noetherian intersections of valuation overrings of two-dimensional Noetherian domains, in preparation.

[34] B. Olberding, The topology of irrredundant intersections of valuation rings, in preparation.

[35] B. Olberding and M. Roitman, The minimal number of generators of an invertible ideal. Multiplicative ideal theory in commutative algebra, 349-367, Springer, New York, 2006.

[36] L. Ruza and J. Vielma, The equality of the patch topology and the ultrafilter topology: A shortcut, Applied General Topology 12 (2011), no. 1, 15-16.

[37] N. Schwartz, Eine universelle Eigenschaft reell abgeschlossener räcme, Comm. Algebra 18 (1990), no. 3, 755-774.

[38] N. Schwartz and M. Tressl, Elementary properties of minimal and maximal points in Zariski spectra, J. Algebra 323 (2010), no. 3, 698-728.

[39] H. W. Schülting, Real holomorphy rings in real algebraic geometry, Real algebraic geometry and quadratic forms (Rennes, 1981), pp. 433-442, Lecture Notes in Math., 959, Springer, Berlin-New York, 1982.

[40] K. Sekiguchi, Prüfer Domain and Affine Scheme, Tokyo J. Math. 13(2) (1990), 259-275.

[41] The Stacks Project Authors, Stacks Project, http://stacks.math.columbia.edu

[42] M. Temkin, On local properties of non-Archimedean spaces II, Isr. J. of Math. 140 (2004), 1-27.

[43] O. Zariski, Foundations of a general theory of birational correspondences, Trans. Amer. Math. Soc. 53, (1943), 490-542.

[44] O. Zariski, The compactness of the Riemann manifold of an abstract field of algebraic functions, Bull. Amer. Math. Soc. 50, (1944), 683-691.

[45] O. Zariski and P. Samuel, Commutative algebra. Vol. II. Graduate Texts in Mathematics, Vol. 29. Springer-Verlag, New York-Heidelberg, 1975. 